\title{
Emission State Structure and Linewidth Broadening Mechanisms in Type-II CdSe/CdTe Core-Crown Nanoplatelets: A Combined Theoretical - Single Nanocrystal Optical Study
}

Violette Steinmetz, ${ }^{\dagger}$ Juan I. Climente, ${ }^{\ddagger}$ Raj Pandya, ${ }^{\llbracket}$ Josep Planelles, ${ }^{\ddagger}$ Florent Margaillan, ${ }^{\dagger}$ Yuttapoom Puttisong,,$\S$ Marion Dufour,, Sandrine Ithurria,,$\|$ Ashish Sharma, ${ }^{\perp}$ Girish Lakhwani, ${ }^{\perp}$ Laurent Legrand ${ }^{\dagger}$ Frédérick Bernardot, ${ }^{\dagger}$

Christophe Testelin, ${ }^{\dagger}$ Maria Chamarro, ${ }^{\dagger}$ Alex W. Chin, ${ }^{\dagger}$ Akshay Rao, ${ }^{\circledR}$ and Thierry Barisien ${ }^{*} \dagger$

$\dagger$ †orbonne Université, Institut des NanoSciences de Paris, INSP, CNRS-UMR 7588, 4 place Jussieu, F-75005, Paris, France

$\ddagger$ Departament de Química Física i Analítica, Universitat Jaume I, E-12080, Castelló de la Plana, Spain

\Department of Physics, Cavendish Laboratory, University of Cambridge, 19 JJ Thomson Avenue, Cambridge, CB3 OHE, UK

§Functional Electronic Materials, Department of Physics, Chemistry and Biology, Linköping University, 58183 Linköping, Sweden

||Laboratoire de Physique et d'Étude des matériaux, ESPCI Paris, PSL Research University, CNRS UMR 8213, 10 rue Vauquelin, 75005 Paris, France $\perp A R C$ Centre of Excellence in Exciton Science, School of Chemistry, The University of Sydney, Sydney, New South Wales 2006, Australia

E-mail: barisien@insp.jussieu.fr 


\begin{abstract}
Type-II heterostructures are key elementary components in optoelectronic, photovoltaics and quantum devices. The staggered band alignment of materials leads to the stabilization of indirects excitons (IXs) i.e. correlated electron-hole pairs experiencing spatial separation with novel properties, boosting optical gain and promoting strategies for the design of information storage, charge separation or qubit manipulation devices. Planar colloidal CdSe/CdTe core-crown type-II nested structures, grown as nanoplatelets (NPLs) are the focus of the present work. By combining low temperature single NPL measurements and electronic structure calculations we gain insights into the mechanisms impacting the emission properties. We are able to probe the sensitivity of the elementary excitations (IXs, trions) with respect to the appropriate structural parameter (core size). Neutral IXs, with binding energies reaching $50 \mathrm{meV}$, are shown to dominate the highly structured single NPL emission. The large broadening linewidth that persists at the single NPL level clearly results from strong exciton-LO phonon coupling $\left(E_{p h}=21 \mathrm{meV}\right)$ whose strength is poorly influenced by trapped charges. The spectral jumps $(\approx 10 \mathrm{meV})$ in the photoluminescence recorded as a function of time are explained by the fluctuations in the IX electrostatic environment considering fractional variations $(\approx 0.2 \mathrm{e})$ of the non compensated charge defects.
\end{abstract}




\section{Introduction}

The preparation of novel heterojunctions achieving a type-II band alignment has raised considerable interest over the past years in the field of colloidal nanocrystals chemistry. ${ }^{1-4}$ In type-II systems the juxtaposition of materials with appropriate staggered band energies leads to an energetic framework allowing the stabilization of indirect excitons (IX) built from spatially separated electron and hole from either side of the hetero-interface present in the structure. This spatial separation of charged carriers has a profound impact on the exciton fundamental properties and has important implications for the development of highly efficient gain media. ${ }^{5,6}$ The reduced overlap between the electron and hole wavefunctions leads to a considerably larger IX radiative lifetime ${ }^{7,8}$ but it was also shown to be responsible for an increase of the multiexciton generation rate $^{9}$ as well as a drastic lengthening of Auger decay times ${ }^{10}$ possibly accompanied by the achievement of strongly repulsive exciton-exciton interaction regimes improving gain properties. ${ }^{5,11}$ An enhancement of the quantum-confined Stark effect was also reported in type-II elongated nanoparticles due to a large permanent dipole accross the interface. ${ }^{12}$ More recently type-II nanostructures have raised a significant interest as they could be used in quantum gates exploiting the controlled interaction of pairs of qubits encoding on neighboring excitons sharing one of their carriers. ${ }^{13}$ Additionally the driving force provided by the band offset between the two materials also allows spontaneous directional charge separation, ${ }^{7,14,15}$ a property that makes type-II systems particularly attractive for photovoltaics.

So far IXs in type-II nanocrystals have been mostly studied in the so called core/shell configuration, one of the material being entirely embedded in the other. In this work we focus on the properties of a less frequently considered cadmium-chalcogenide-based structure characterized by a different topology. The lateral growing of a CdTe shell around a parallelepipedic core made of CdSe provides core-crown nanoplatelets of uniform few monolayers thickness

and atomic level controlled flatness that combine two advantages: ${ }^{16-19}$ a well marked type-II band alignment and, due to the high degree of transverse - along thickness - confinement, 
an ehancement of the IX binding energy leading to an increased robustness against temperature. Whereas most studies have focused on the IX formation dynamics and multiexcitonic relaxation properties in this type of heterostructure ${ }^{17,18,20,21}$ little is known about the band edge IX itself and its coupling properties to the environment (including phonons and electrostatic interactions). Here, using low temperature micro-photoluminescence (microPL) spectroscopy, we address the emission properties of individual CdSe/CdTe core-crown nanoplatelets (NPLs). We gain insights into the microscopic mechanisms determining the nature and influencing the structure of optical excitations. The PL of individual NPLs exhibits a highly structured atypically broad spectral lineshape. On the basis of our calculations and by varying a structural parameter (core size) we are able to reject several assumptions concerning the origin of the multipeaked emission. In particular, we show that the emission quasi-exclusively corresponds to the recombination of the lowest energy IX state. Our results point to a major role of the exciton-phonon coupling onto the homogeneous lineshape broadening. The impact of the dielectric contrast between the NPL and the outside medium on the IX structure and energy is also discussed. The observation of spectral jumps occurring on the seconds time scale in the single NPL PL leads us to investigate the influence of charged defects upon the fundamental excitonic transition (zero phonon line - ZPL) and its vibronic replicas. We quantify the ZPL energy perturbation induced by non compensated charges as a function of their position in the structure and qualitatively show how additional charge may, by changing the symmetry of the whole charge distribution (electron, hole and defects) reinforce vibronic coupling. Our work provides an unified description of how the microscopic coulombic mechanisms (through dielectric confinement and charge trapping) and exciton-phonon coupling combine and interfere to shape the optical response of core-crown type-II heterostructures. 


\section{Experimental results}

In order to gain insights into the NPLs excited states structure, NPLs having distinct core sizes were considered in this work. Large core (LC) NPLs were synthesized with typical dimensions of the core and crown being respectively $\approx 37 \times 10 \mathrm{~nm}^{2}$ and $\approx 65 \times 18 \mathrm{~nm}^{2}$. In small core $(\mathrm{SC})$ NPLs the core size was lowered to $\approx 12 \times 11 \mathrm{~nm}^{2}$ and the crown to core surfaces ratio was roughly maintained compared to the LC NPLs (crown size $\approx 33 \mathrm{x} 19 \mathrm{~nm}^{2}$ ). The NPLs thickness was kept constant in the two batches (4 monolayers (MLs)) with a growth engineering ensuring atomic flatness when moving from the core to the crown domains (see the Methods section for description of synthesis and SI, S1 for TEM images). Figure 1a shows the $10 \mathrm{~K}$ absorption and PL spectra of LC NPLs prepared as thin solid films, deposited from a moderately diluted solution. The absorption exhibits the characteristic features of type-II CdSe/CdTe hetero-NPLs with a broad tail expanding on the low energy side of a well marked edge at $550 \mathrm{~nm}\left(E_{d} \approx 2.26 \mathrm{eV}\right)$. It is now well established that the $\mathrm{X}_{i}, i=1-4$ transitions evidenced as sharp peaks above $E_{d}$ can be assigned to the absorption from excitons formed from the electron-heavy-hole (e-hh) and electron-light-hole (e-lh) pairs of both the CdSe and CdTe bare materials. ${ }^{16,17,21-23}$ In the present study we observe $\mathrm{X}_{1}$ (e-hh transition) and $\mathrm{X}_{3}$ (e-lh transition) of the CdTe crown at $2.33 \mathrm{eV}$ and $2.58 \mathrm{eV}$ respectively whereas cor-

responding transitions, $\mathrm{X}_{2}$ and $\mathrm{X}_{4}$, for the CdSe core are measured at $2.54 \mathrm{eV}$ and $2.67 \mathrm{eV}$. We note in particular that the spectral shifts experienced by the $\mathrm{X}_{i}$ lines between ambient temperature and $T=10 \mathrm{~K}(\Delta \approx 100 \mathrm{meV})$ are well reproduced using the Varshni semiempirical relation explaining the bandgap behaviour versus T (see Supporting Information). 

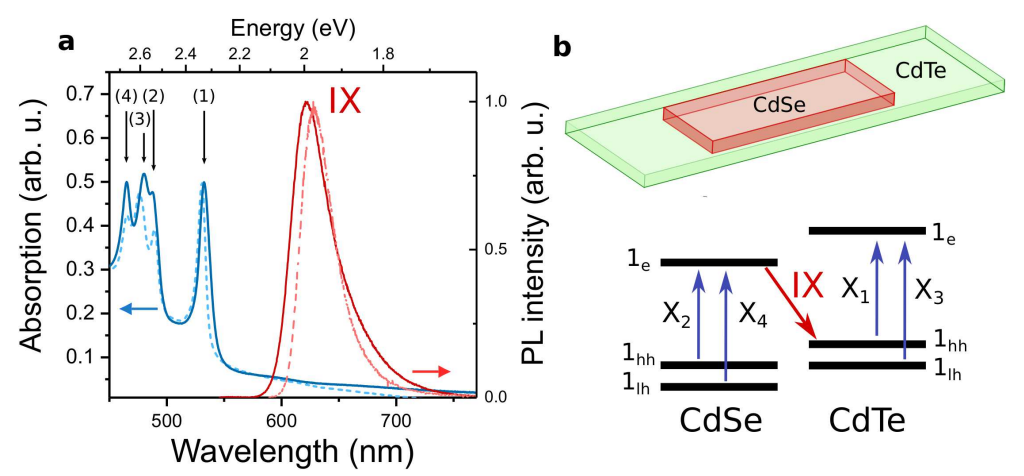

Figure 1: (a) Absorption and photoluminescence (PL) spectra of a dense film of core-crown CdSe/CdTe NPLs at low temperature $(\approx 10 \mathrm{~K})$ : Large Core (LC) NPLs, solid lines and Small Core (SC) NPLs, dash lines. (b) Schematic of the core-crown CdSe/CdTe NPLs geometry and diagram of the single carriers energy levels.

Following excitation at energies above $E_{d}$ the photogenerated excitons in both the CdSe and CdTe domains are dissociated across the CdSe/CdTe interface despite their large binding energies $\left(100-200 \mathrm{meV}^{24-27}\right)$. Although still debated, the dissociation dynamics is fast and leads to an exciton with spatial separation of its charges, an indirect exciton (IX), also sometimes named charge-transfer exciton, within less than a picosecond. ${ }^{17,21}$ As a consequence the CdSe and CdTe PL can only be observed transiently, ${ }^{20,21}$ and the NPL PL is finally dominated by the recombination of the IX which signature consists of a strongly red-shifted band centered at $620 \mathrm{~nm}$ or $\approx 2 \mathrm{eV}$ (Figure 1a, red curve and energy levels, Figure 1b). As mentioned in previous works, this emission band is surprisingly broad despite the utmost control of the confinement direction during the growth process ${ }^{16}$ leading to a quasi-absence of size dispersion associated to this dimension. A full width at half maximum (FWHM) of $140 \mathrm{meV}$ at $10 \mathrm{~K}$ is reported here and should be compared to the direct $\mathrm{X}_{1}$ transition width in absorption, $\approx 5$ times smaller. It is well known that IX in type-II systems experience much larger line broadening for they feel the potential inhomogeneities associated to the interface imperfectness. ${ }^{28}$ Previous works have highlighted the highly ordered nature of the core/crown interface ${ }^{21}$ and it was moreover claimed that owing to the level of structural quality (in particular in absence of dispersion in thicknesses), inhomogeneous effects could 
not exclusively be held responsible for the broadening in this system. ${ }^{16}$ The same conclusion was drawn in other studies considering high quality type-II colloidal nano-heterostructures. ${ }^{4}$ The point is discussed later on, further insights being first gained by the inspection of the single NPLs PL response.

The IX core-crown NPLs was also shown to be a weakly absorptive excitation, a property already reported for analogous type-II cadmium chalcogenide based nanostructures. ${ }^{29,30}$ Study of the ground state bleach dynamics between 540 and $700 \mathrm{~nm}$ and PL excitation experiments ${ }^{17,21}$ both led to the conclusion that a weak oscillator strength (a few percents of that of the CdTe excitons) is associated to the well visible broad tail expanding below $E_{d}$ which excitation resulted in the IX formation. Direct excitation of IX species was indeed found useful to investigate the IX fine structure properties in magneto-PL experiments performed under quasi-resonant excitation conditions. ${ }^{31}$ Finally, at this point, LC and SC NPLs absorption and PL do not differ substantially (Figure 1a). There are minor differences in the transitions energies (a few meV typically) and oscillator strengths associated to the CdSe and CdTe domains ( $\mathrm{X}_{i}$ transitions) as the size varies. The normalization of the $\mathrm{SC}$ and LC NPLs absorption at the $\mathrm{X}_{1}$ transition energy (CdTe crown exciton) indicate a smaller weight of the CdSe domain response in SC NPLs $(\approx 1.4$ times less $)$ in agreement with their larger crown to core surface ratio (this ratio is $\approx 1.7$ larger in SCs NPLs). The point will not be discussed further here. We note that the similarity in the absorption seems consistent with the situation of systems sharing moderate lateral confinement but identical and relatively strong transverse confinement (that for comparable environments will govern single particles energies, exciton binding and self-interaction effects). ${ }^{25,27}$ By contrast a larger sensitivity to the crown to core dimensions ratio is expected for the IX transition whose properties are determined by the electron and hole wavefunctions overlap across the interface. In this regard we notice a significant PL redshift $(\approx 25 \mathrm{meV})$ in smaller core NPLs. 

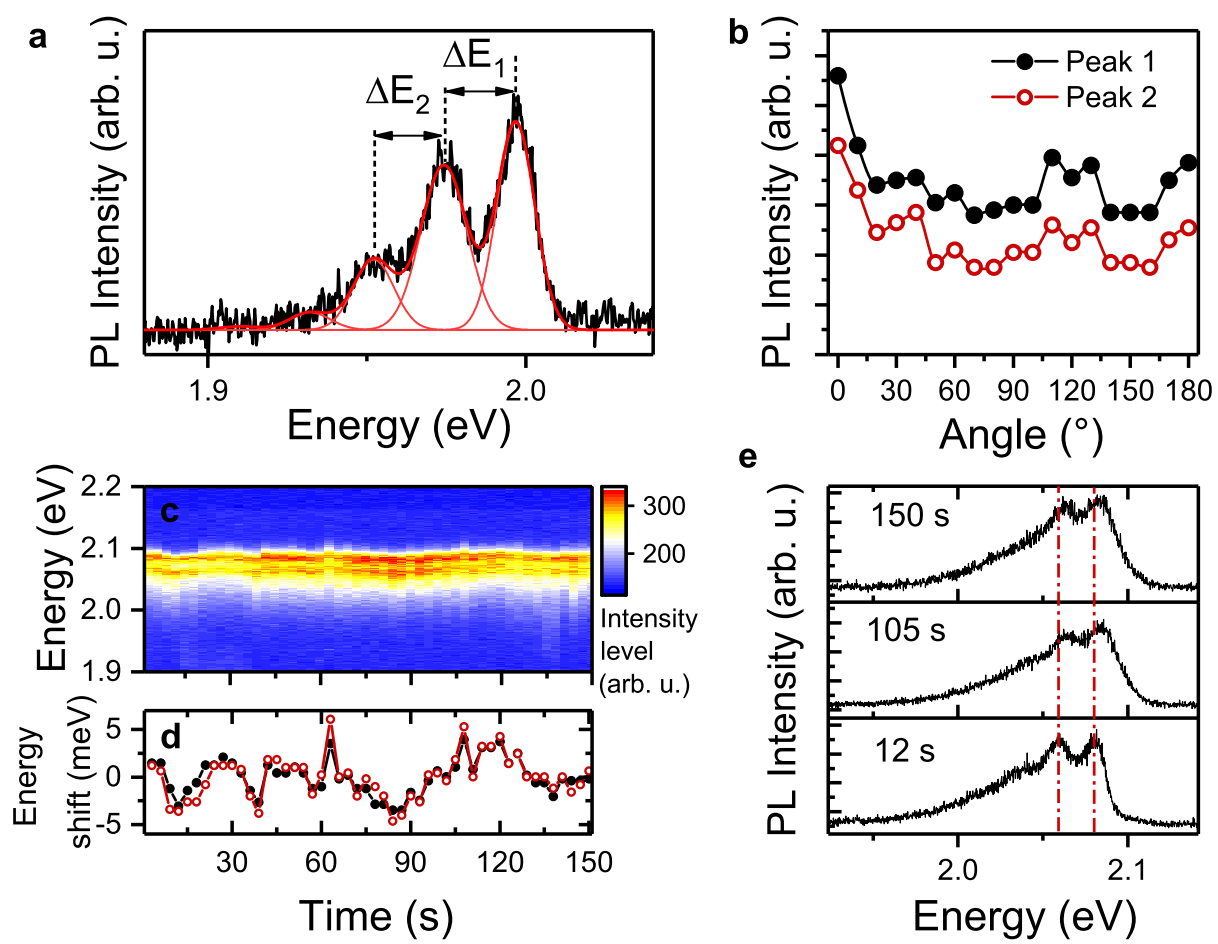

Figure 2: (a) Typical PL spectrum of a single NPL (excitation at $532 \mathrm{~nm}$, c.w., incident power equal to $1.3 \mathrm{~kW} / \mathrm{cm}^{2}$ ) and associated multicomponent fit leading to a Huang-Rhys factor $S \approx 0.8$. (b) Polarization analysis of the emission associated to the higher energy peak (peak 1) and to its first satellite (peak 2); the solid lines are a guide to the eye. The scan is obtained by rotating a $\lambda / 2$ waveplate in the plane of the sample and using a polarizer with fixed axis (serving for the polarization analysis) in front of the spectrometer slits. (c) Emission of another single NPL recorded as a function of time (2 seconds integration time per spectrum). (d) Associated peak 1 and peak 2 energy displacement. (e) Sampling of the emission (spectrum) at given time position. The vertical dash-dot lines indicate the peak positions at $\mathrm{t}=12 \mathrm{~s}$.

In order to have access to single NPLs properties, films obtained from highly diluted solutions (concentration lowered up to 2000 times from the original solution) were considered. The studies were found to suffer from relatively poor photo-stability of the individual NPLs. The power density was thus kept as low as a few $\mu \mathrm{W} / \mu \mathrm{m}^{2}$ to avoid deterioration. Under those conditions and taking into account the moderate numerical aperture of the objective $($ N.A. $=0.65)$ a reasonable signal to noise ratio was typically reached for an integration time being no less than one second, preventing any clear tracking of the NPL emission at a shorter timescale. Figure 2a shows the typical emission from a single LC NPL measured 
at $5 \mathrm{~K}$ as the excitation (continuous wave laser) is tuned just above the IX band edge ie onto the $\mathrm{X}_{1}$ transition $\left(\lambda_{e x} \approx 533 \mathrm{~nm}\right)$. The spectrum reveals several evenly-spaced peaks (energy separation $\Delta \mathrm{E} \approx 20 \mathrm{meV}$ ) decreasing in intensity as their energy decreases. In most spectra one satellite peak is clearly visible with a lower energy part that consists of a more structureless tail. A regular progression can however extends over one hundred meV and reveal up to three replicas, as in the spectrum shown in Figure 2a that is satisfactorily adjusted using a multi-Gaussian profile.

In comparable experimental conditions CdSe homoplatelets exhibit markedly different characteristic PLs: the linewidths are found around $400 \mu \mathrm{eV}$ while the PL intensity is typically found two hundred times higher (not shown). We conclude that the area below the PL spectra is roughly the same in each type of NPL and that both types have similar emission yields. This result is consistent with previous observations in crown alloyed NPLs ${ }^{32}$ and the report of both the quasi-complete conversion of the photoexcitations towards IXs and a high IX PL quantum yield $(\approx 86 \%)$ at $4 \mathrm{~K}$ in pure CdSe/CdTe core-crown NPLs. ${ }^{31} \mathrm{~A}$ typical value of the residual broadening affecting the PL peaks, and in particular the lowest energy emission line, is given by the full width at half maximum (FWHM) extracted from the fit $\left(\Delta E_{p k} \approx 15-20 \mathrm{meV}\right)$. The emission spectrum as a function of time is presented in Figure 2c for another NPL (same conditions of excitation), each spectrum being collected with a 3 s exposure time. Despite strong intensity fluctuations the series clearly show instances of spectral diffusion characterized by a rigid shift of the spectrum, the latter very likely resulting from spontaneous modification of the electrostatic environment through charging or discharging of the individual NPLs. ${ }^{33}$ Over long range time scale, shifts of $\approx 7 \mathrm{meV}$ amplitude can clearly be evidenced (Figure $2 \mathbf{d}$ ). They will be related to the Stark-effect mediated electrostatic fluctuations (combined to the type-II nature of the exciton) in the next section where supporting complementary calculations are carried out in order to assess the impact of IX - trapped charges interactions. In addition, given the used integration time in the experiment $(1-3 \mathrm{~s})$, each snapshot shows that the peaks composing the PL remain 
themselves largely broadened, their linewidth $\Delta E_{p k}$, being comparable to the previously reported shifts. It is noteworthy that $\Delta E_{p k}$ is already $\approx 100$ times larger than the typical linewidths extracted in 'classical' spectrally resolved micro-PL experiments performed on chalcogenides nanocrystals and that are often qualified as homogeneous widths. ${ }^{32-34}$ We believe that such broadening effects are also extrinsic and of electrostatic nature ${ }^{35}$ and that they may be the result of faster charge fluctuations. Given the poor signal to noise ratio in single NPLs measurements the point could not be explored further, simply be decreasing the integration time.

Note that the emission pattern of a single NPL (interpeak distance and relative amplitudes) remains unchanged in any of the PL(t) records. This is in contradiction with peaks that would originate from Auger-assisted recombination in which an electron is promoted to a higher energy level and a photon emitted at an energy below the exciton gap. Along the spatial coordinates the confinement potential seen by electron and holes indeed varies abruptly and rectangular potentials cannot explain a regular spacing of the upper energy levels, the latter being instead specific to an electronic coupling driven by a harmonic oscillator i.e. the nuclear motion. ${ }^{36}$ We are thus led to discard any shake-up like mechanism, that would be at play and responsible for the multiplication of peaks in the emission. ${ }^{37}$ The regular energy spacing discards as well simultaneous emission from different states within a stack of NPLs. ${ }^{38}$ Further experimental evidences are given below. The actual single particles (electron and hole) energies are also determined as a function of the NPL core size in the Numerical Simulations section and compared to the measurements.

In CdSe/CdTe core-crown NPLs the exciton fine structure consists of two bright states emitting with linear crossed polarizations aligned along the NPL length and width dimensions. ${ }^{31}$ The fine structure splitting $(\approx 60 \mu \mathrm{eV})$ is almost two orders of magnitude smaller than (1) the FWHM reported here for the peaks appearing in the PL spectrum, (2) the inter-peak energy distance $\Delta E$. For a non-resonant excitation and assuming that the exciton transitions have similar oscillator strengths, one should thus expect that the polarization 
diagram of a single NPL only reflects shape anisotropy that, through band edge symmetry ${ }^{39}$ and internal field effects (resulting from the strong dielectric confinement), ${ }^{40-42}$ will favor the transition associated to the larger lateral dimension. The application of classical formula in an ellipsoid model show that a factor $\approx 2$ should be expected in the intensity radiated by the exciton sublevels for isolated LC NPLs deposited on a glass substrate in air (the ratio falls to $\approx 1.5$ in SC NPLs), and that it is a priori experimentally reachable (see Supporting Information). Nevertheless large PL intensity fluctuations during the angle scan prevent the observation of the anisotropy of the polarisation analysis (Figure 2b). The measurements however show that peaks composing the spectrum all share the same polarization properties demonstrating that lower energy peaks correspond to composite excitations formed from the fundamental exciton. Consequently the observed structures with regular energy progression can unambiguously be assigned to vibronic replicas associated to a single NPL response.
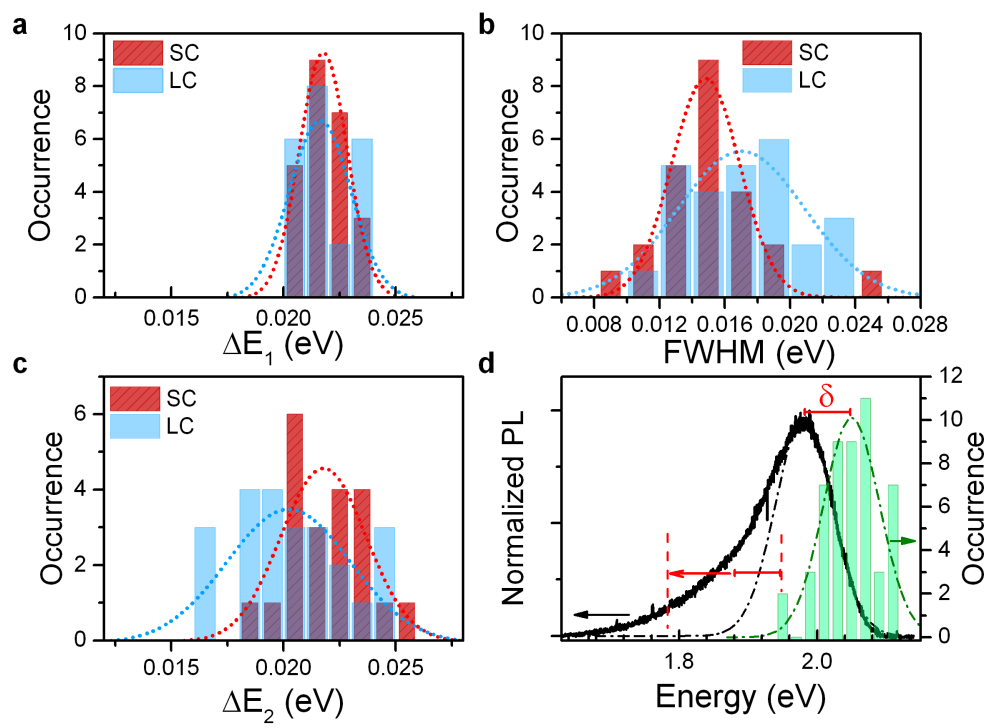

Figure 3: Histograms for the interpeak energy distance, $\Delta E_{1}$ (a) and $\Delta E_{2}$ (c), zero phonon line (ZPL) linewidth (b) and ZPL transition energy, green bars (d). The dot lines are Gaussian fits of the distributions (a-c) in small core (SC) and large core (LC) NPLs. In (d) the spectral extension of the inhomogeneous PL (solid line) is compared to the broadening that would be expected when shifting the ZPL energy distribution from $\delta$ (arising from dielectric effects) and adding a vibronic contribution of $\approx 4 \times \Delta E_{1}$ (red arrow). In the study $\delta=69 \pm 10 \mathrm{meV}$ in agreement with numerical estimations (see next section). 
To build a more representative picture of the system properties, data originating from a panel of 25 individual objects were collected and a basic statistical analysis was performed. Corresponding histograms are gathered in Figure 3 for the inter-peak energy distances, the zero phonon line (ZPL) FWHM and ZPL energy. We emphasize that the ZPL energy distribution (and associated satellites) is deduced from snapshots (corresponding to low integration times measurements) but remains representative due to the random nature of the spectral shift mechanism. Adjustments using a normal distribution provides the mean values as well as their standard deviations. Noting $\Delta E_{i}, i=1,2$ the energy separation between the first and second and second and third peaks in the spectra, respectively, we find that the $\Delta E_{i}$ can be considered as independent of the NPL size. In LC (respectively SC) NPLs the adjustment provides $\Delta E_{1}=22 \pm 2 \mathrm{meV}\left(\Delta E_{1}=22 \pm 1 \mathrm{meV}\right)$ and $\Delta E_{2}=20 \pm 3$ $\operatorname{meV}\left(\Delta E_{2}=22 \pm 2 \mathrm{meV}\right)$. As a first approach, the values are in good agreement with the volume average between the LO-phonon frequencies of bulk CdTe (21 meV) and bulk CdSe $(26 \mathrm{meV}),{ }^{43}$ found equal to $22.5 \mathrm{meV}$ in LC NPLs $(31 \% \mathrm{CdSe}, 69 \% \mathrm{CdTe})$ and $22.1 \mathrm{meV}$ in SC NPLs (21 \% CdSe, 79\% CdTe). In good agreement a strongly active mode shows up in the low temperature Raman spectrum of a moderately diluted sample with a Stokes band centered at $166 \mathrm{~cm}^{-1}$ ie corresponding to an optical phonon energy of $\approx 20.6 \mathrm{meV}$ (see SI, S3). Our data also seem to point a slight increase of the ZPL FWHM (from $15 \pm 2 \mathrm{meV}$ to $17 \pm 4 \mathrm{meV}$ ) as the core size increases.

The Huang-Rhys factor, $S$, quantifies the amount of oscillator strength that is borrowed to the ZPL by each of the phonon-assisted transitions. In the displaced oscillators model the $\mathrm{n}^{\text {th }}$ phonon replica should then have a weight proportional to $\exp (-S) S^{n} / n$ ! (see SI). A careful analysis shows that most of the recorded single NPL spectra have a vibronic structure that indeed exhibits the appropriate dependence with n, the $S$ values ranging from 0.6 to 1.6. We notice that $0.8 \leq \mathrm{S} \leq 1.0$ is typical of $\approx 80 \%$ in number of the studied NPLs (Figure $\mathbf{2 a}$ and Supporting Information). Within the available statistics, the distribution of the $S$ factor values were found independent of the NPL core size. 
Finally we find that individual ZPL energies are distributed within a relatively narrow band $\left(\gamma_{i n h} \approx 80 \mathrm{meV}\right.$ in width) that reasonably mimics the high energy part of the PL inhomogeneous (ensemble) spectrum. The centre of this distribution is shifted from the top of the inhomogeneous PL by an amount $\delta$. The average $\delta$ value is found equal to $69 \pm$ $10 \mathrm{meV}$. When accounting for the low energy wing induced by the vibronic coupling (see Figure 3d) the extension of the ensemble emission can be rebuilt and is found to nicely match the experimental PL band. We show in the next section that the marked blueshift, $\delta$, of the reconstructed distribution can reasonably be explained by accounting for the actual dielectric environment of an isolated NPL.

\section{Numerical Simulations}

We can gain understanding of the spectroscopic results in the previous section by analyzing the underlying electronic structure. To this end, we perform effective mass calculations of carriers confined in CdSe/CdTe NPLs like that of Figure 4a. We take crown dimensions $18 \times 40 \mathrm{~nm}^{2}$ and core dimensions $10 \times L_{y}^{c} \mathrm{~nm}^{2}$, where the core length $L_{y}^{c}$ is varied from 10 to $30 \mathrm{~nm}$, thus covering the bridge between our SC and LC NPLs. Figure $\mathbf{4 b}$ shows the energies of the lowest conduction band electron states (top panel) and highest valence band hole states (bottom panel). Each color indicates the symmetry (irreducible representation) of the state, within the $D_{2 h}$ point group of the NPL. The correspondence between colors and symmetry is given in Figure 4c, which further illustrates the envelope function of the first electron and hole states of each symmetry. 

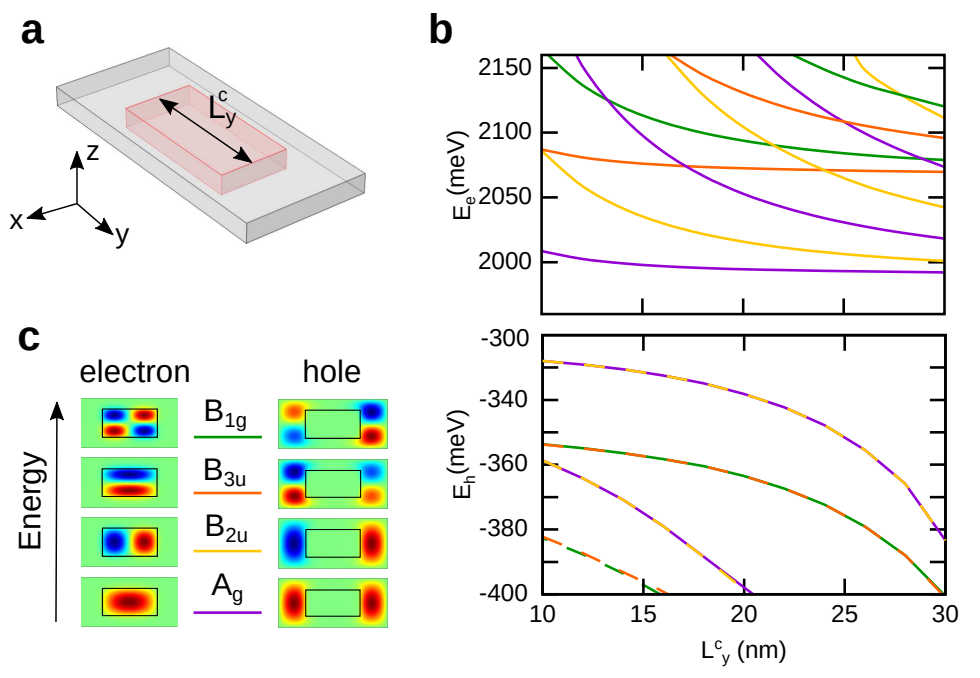

Figure 4: (a) Geometry of the core-crown NPL model. The core length $L_{y}^{c}$ is varied, while all other dimensions are fixed. Point group is $D_{2 h}$. (b) Electron (top, solid lines) and hole (bottom, dashed lines) independent particle energies vs core length. Each color denotes different irreducible representation in $D_{2 h}$ point group. Energies are referred to the top of the valence band. (c) Wave function of lowest electron (left) and highest hole (right) states with each symmetry. The color line corresponds with that in (b).

It is clear from the figure that electrons and holes present different behaviours. The former are confined inside the CdSe core and closely resemble particle-in-2D-box states. As the core length increases, the energy spectrum evolves towards that of a 1D wire, where states with the same number of nodes along $y$ tend to converge. Holes in turn are confined in the CdTe crown. Because the crown is narrow along the $x$ direction, with the core introducing a repulsive potential, hole states form two-fold degenerate solutions of a double quantum well along $y$ ( $A_{g^{-}} B_{2 u}$ vs $B_{1 g^{-}} B_{3 u}$ doublets). Contrary to electrons, increasing the core size introduces quantum confinement for holes and their energy increases.

The above results show a clear charge separation of electrons and holes at an independent particle level, driven by the type-II band offset of CdSe/CdTe. The question arises of whether it persists for excitons, since Coulomb attraction is very strong due to the dielectric confinement organic ligands impose on NPLs. ${ }^{25,27,44}$ We then calculate exciton states using a Configuration Interaction method, with full inclusion of dielectric effects (see Methods). 

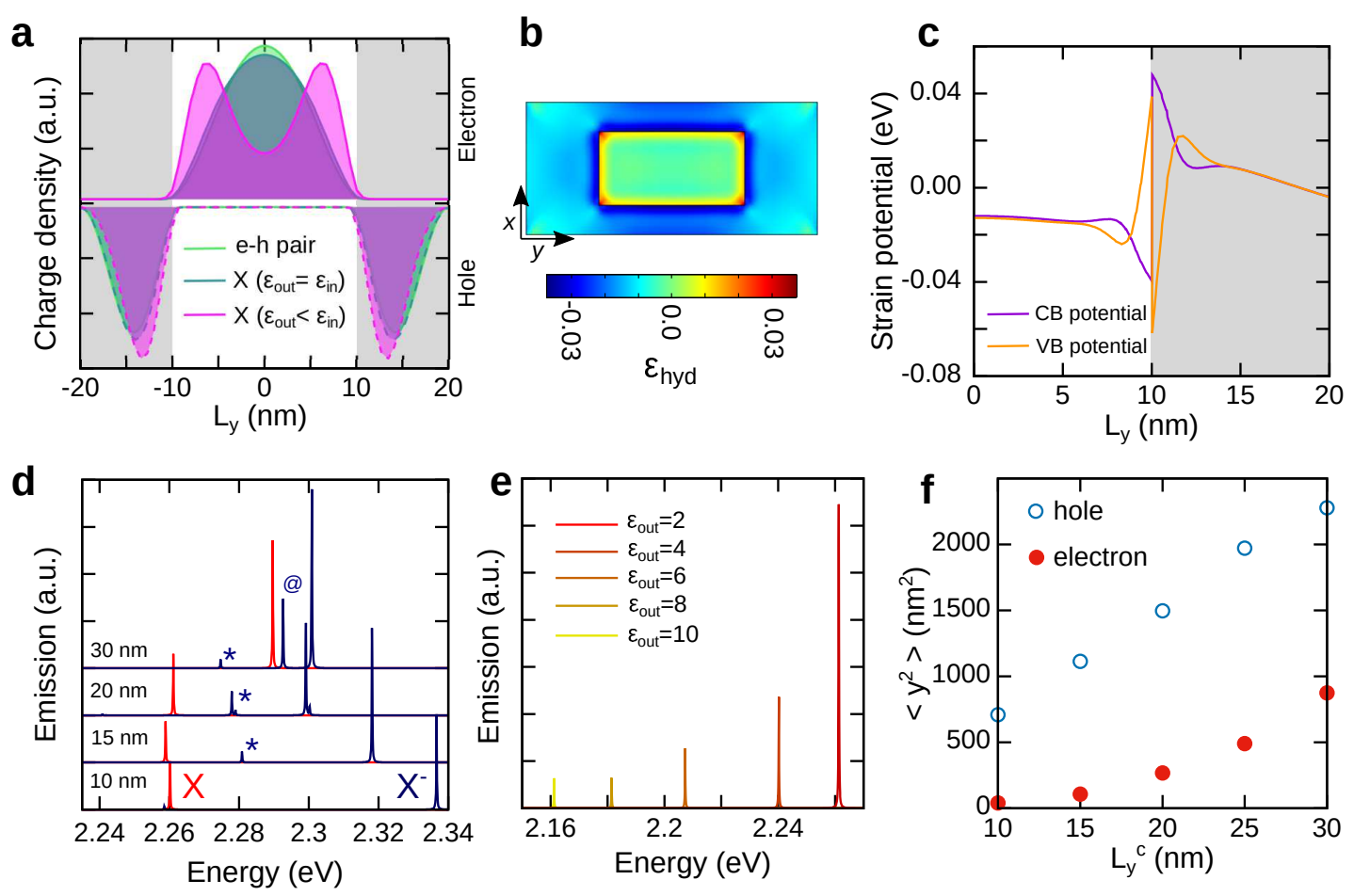

Figure 5: Calculated excitonic properties. (a) Exciton ground state charge density crosssection for a NPL with $L_{y}^{c}=20 \mathrm{~nm}$. The grey-shaded region corresponds to the crown. (b) Hydrostatic strain on the $x y$ plane of the NPL. (c) Deformation potential ensuing from the strain near the core/crown interface for conduction and valence bands. (d) Emission spectrum at $4 \mathrm{~K}$ for excitons $(\mathrm{X})$ and negative trions $\left(\mathrm{X}^{-}\right)$, as a function of the core length. Stars indicate trion shake-up replicas, @ indicates emission from a triplet state. (e) Exciton emission dependence on the outer medium dielectric constant. (f) Mean value of $y$ coordinate for exciton's electron and hole as a function of the core length.

In Figure 5a we compare the ground state charge density along the long axis of a NPL with $L_{y}^{c}=20 \mathrm{~nm}$, which is an intermediate value between the SC and LC NPLs. Three different degrees of Coulomb interaction are considered: (i) non-interacting e-hh pair (pale green curve), (ii) interacting exciton, but neglecting dielectric mismatch (i.e. setting the environment dielectric constant equal to that of the NPL, $\varepsilon_{\text {out }}=\varepsilon_{\text {in }}=10$, dark green curve), and (iii) interacting exciton, considering dielectric mismatch $\left(\varepsilon_{\text {out }}=2<\varepsilon_{\text {in }}=10\right.$, pink curve). In all three cases the ground state has a clear indirect nature, consistent with Figure 1a. This is because the staggered band offset potential ( $0.53 \mathrm{eV}$ for electrons, $-0.69 \mathrm{eV}$ for holes) overcomes the exciton binding energy $(\sim 0.15 \mathrm{eV}$ inside CdSe or $\mathrm{CdTe})$. It also overcomes 
the potential wells that lattice mismatch strain induces on the core/crown interfaces. As shown in Figure 5b, strain is moderately tensile/compressive on the CdSe/CdTe side of the interface. This leads to wells of tens of meV depth near the interface, as illustrated in Figure 5c. The wells are however too narrow to have a significant influence even on the non-interacting e-hh pair, for all core sizes we have studied.

The effect of dielectric mismatch is nevertheless noticeable. When $\varepsilon_{o u t}=\varepsilon_{i n}$, the exciton electron is in the center of the core, while the hole is centered in the crown wings, similar to the non-interacting particles. By contrast, when $\varepsilon_{\text {out }}<\varepsilon_{\text {in }}$, the carriers shifts towards the core/crown interface. This is because dielectric confinement enhances the IX binding energy from $10 \mathrm{meV}$ to $50 \mathrm{meV}$, which is comparable to the values in type-I CdSe quantum dots. This finding supports the conclusions of earlier experiments, which suggested that the short emission lifetime of CdSe/CdTe NPLs must be ascribed to IX bound across the interface, rather than ionized e-hh pairs. ${ }^{45}$

In Figure 5d we plot the calculated emission spectrum of neutral (red line) and negatively charged (blue line) excitons, for different core lengths. The behaviour is clearly different for each species. When decreasing the core length, excitons show a moderate redshift and trions a blueshift instead. The different response originates in the relevant Coulomb terms in each case. Decreasing the core size increases e-hh attraction, which contributes to redshift the exciton ground state, but it also enhances repulsive electron-electron repulsion in the trion. Because the two electrons are localized in the core, their Coulomb interaction is much stronger than that with the crown hole. As a consequence, the repulsion prevails over attraction and trions experience a pronounced blueshift. From the above results, one can note that the redshift of SC as compared to LC NPLs in Figure 1a is indicative of emission stemming from neutral excitons rather than trions. Dual emission from exciton and trion ground states, which has been reported in core-only CdSe NPLs, ${ }^{46,47}$ does not seem to be relevant in CdSe/CdTe NPLs. As shown in Figure 5d, the energy spacing between exciton and trions in type-II systems should rapidly decrease with $L_{y}^{c}$. While we observe 
multi-peaked emission in PL measurements, the energy spacing is similar for SC and LCs, see $\Delta E_{1}$ in Figure 3a.

Trion emission in Figure 5d shows low-energy replicas (marked with stars), which increase in intensity and number as the core length increases. These are so-called shake-up lines, which occur when part of the trion radiative recombination energy is spent on exciting the remaining electron. Such processes are relevant in the weak confinement regime, and have been recently reported in large NPLs. ${ }^{37}$ However, it should be noted that shake-up lines are not energetically iso-spaced (see $L_{y}^{c}=30 \mathrm{~nm}$ graph), and hence cannot be held responsible for the multi-peaked emission in Figure 2a. As anticipated in the previous section, regular (iso-spaced) energetic progress is not expected in NPLs because the electron is described by particle-in-box states, which need not have constant energy spacing, see Figure 4b. The peak marked with @ for $L_{y}^{c}=30 \mathrm{~nm}$ corresponds to a triplet trion state, which for large NPLs is thermally occupied at $4 \mathrm{~K}$. By comparing the theoretical estimates with our experimental results, it is clear that both the $L_{y}^{c}$ dependence of the emission energy and the absence of irregularly spaced (shake-up or triplet) peaks suggest CdSe/CdTe NPLs are charged with neutral excitons, rather than trions.

We next turn to the blueshift systematically observed in the PL of diluted NPLs, as compared to high concentration ensembles, $\delta$ in Fig. 3d. Similar concentration dependent shifts have been reported in $\mathrm{CsPbBr}_{3} \mathrm{NPLs}$, and were partially ascribed to re-absorption and partially to changes in the dielectric environment. ${ }^{48}$ Re-absorption can be ruled out in our system because the type-II band structure largely suppresses the overlap between absorption and emission, as shown in Figure 1a. The concentration of NPLs however is susceptible of varying the average dielectric constant of the environment seen by an individual NPLs. As the concentration and proximity between NPLs increases, the dielectric constant should evolve from typical organic environment values, $\varepsilon_{\text {out }} \approx 2$, to values ultimately approaching CdSe or CdTe constants, $\varepsilon_{\text {out }} \approx 10$. Figure 5e shows the calculated evolution of the exciton emission as a function of $\varepsilon_{\text {out }}$. Two clear effects of dielectric confinement are observed with 
decreasing $\varepsilon_{\text {out }}$ (increasing NPLs dilution). Namely, the emission peak gains intensity and 'blueshifts'. The first effect is due to the reduced exciton Bohr radius, which translates into faster interband recombination rate. ${ }^{49}$ The second is a consequence of the (repulsive) selfenergy potential outweighing (attractive) e-hh Coulomb enhancement, ${ }^{25}$ which is especially pronounced in type-II structures. ${ }^{27}$ It follows from the figure that an upperbound blueshift of $100 \mathrm{meV}$ may be explained by NPL dilution. The smaller value in the experiments, $\delta \approx 70$ meV in Fig. 3d, can be interpreted as the effective dielectric constant varying between $\varepsilon_{\text {out }} \sim 2$ in diluted NPLs to $\varepsilon_{\text {out }} \sim 6$ in concentrated ones. $\varepsilon_{\text {out }}$ does not reach higher values because organic ligands remain on the surface even for high NPL concentrations. 

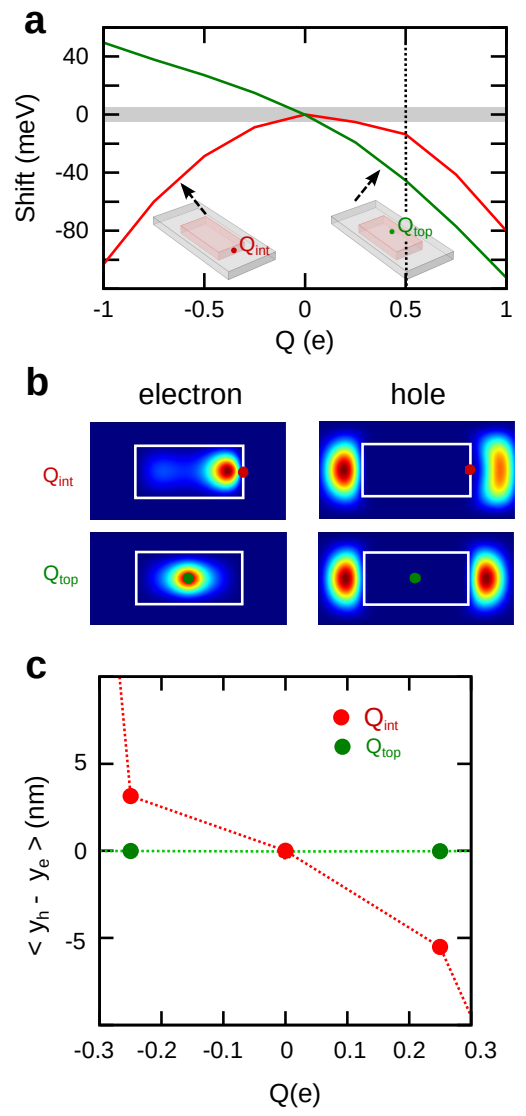

Figure 6: Effect of a point charge on the exciton transition energy and wavefunctions. (a) Exciton energy shift induced by a point charge. Two different charge locations are considered. $Q_{\text {int }}$ (red line) is in the middle of the core-crown interface along $y$. $Q_{t o p}$ is in the center of the core, $1.7 \mathrm{~nm}$ above the surface. Charge strength is varied from fully unscreened electron $(-e)$ to fully unscreened hole $(+e)$. $e$ is the fundamental charge. (b) Effect of a 0.5e point charge on the excitonic electron and hole charge densities. (c) Mean value of the electronhole spacing along coordinate $y$ as a function of charge added in the 'on-top' and 'interface' positions.

To assess on the origin of the spectral shifts reported in Figure 2c, we also calculate the exciton ground state energy in the presence of a trapped carrier, acting as a point charge potential. Trapping sites can be expected on the NPL surface or core-crown interface, in principle to either electrons or holes. We account for these possibilities by modeling a point charge centered on the top surface of the NPL, and another on the core-crown interface, each with varying charge strength and sign. Figure $\mathbf{6 a}$ shows the energy shift they induce on the exciton energy. One can see the response is very different. The charge centered in the core 
( $Q_{\text {top }}$, green line) has an asymmetric response for positive and negative values of the charge. By contrast, the interface charge $\left(Q_{\text {int }}\right.$, red line $)$ is more symmetric, and has an overall weaker influence. The different behavior can be easily understood from Figure 6b, which visualizes the effect of a fractional positive point charge $(Q=0.5)$ in each location over the exciton's electron and hole charge densities. Being in between the two carriers, $Q_{\text {int }}$ respectively attracts and repels the electron and the hole to a comparable extent. Conversely, $Q_{t o p}$ has a strong influence on the core electron but not the crown hole. The lack of compensation leads to a more conspicuous response.

The spectral shifts reported in the experimental section lie in the $\pm 5 \mathrm{meV}$ range. This region is highlighted with a grey-shaded area in Figure 6a. Clearly, the experimental response corresponds to that of fractional charges, $|Q|<0.2 e$. This is consistent with atomistic calculations in CdSe quantum dots, which note that realistic trapped charges are significantly screened as compared to full point charges. ${ }^{50}$

The exciton states we have calculated can also provide some information on the influence of the core-crown geometry in determining the coupling to LO-phonons. The Huang-Rhys factor $(S)$ for an exciton state $\Psi_{\mu}$ is proportional to exciton-phonon coupling terms of the form: $:^{51}$

$$
v_{\mu \mu}(\mathbf{k})=\int d^{3} r_{e} d^{3} r_{h} \Psi_{\mu}^{*} \Psi_{\mu}\left[\exp \left(i \mathbf{k r}_{\mathbf{e}}\right)-\exp \left(i \mathbf{k r}_{\mathbf{h}}\right)\right]
$$

where $\mathbf{k}$ is the phonon wave vector and $r_{j}(j=e, h)$ the position of electron and hole. The phonon plane waves in the above equation can be Taylor-expanded. Upon integration and in the absence of trapped charges, the dipolar terms of the expansion, $\mathbf{k r}_{j}$, vanish because of the odd symmetry of $\mathbf{r}_{j}$. The lowest term of the expansion is then quadrupolar,

$$
v_{\mu \mu}(\mathbf{k}) \approx-\frac{1}{2} \int d^{3} r_{e} d^{3} r_{h} \Psi_{\mu}^{*} \Psi_{\mu}\left[\mathbf{k} \mathcal{R}_{e} \mathbf{k}-\mathbf{k} \mathcal{R}_{h} \mathbf{k}\right]
$$

where $\mathcal{R}_{j}$ is the polarization tensor. The most relevant term in the tensor is that along the 
long axis of the NPL, $y^{2}$. We can then infer the variation of $S$ from:

$$
v_{\mu \mu}(\mathbf{k}) \approx \frac{1}{2} k_{y}^{2} \int d^{3} r_{e} d^{3} r_{h} \Psi_{\mu}^{*} \Psi_{\mu}\left[y_{h}^{2}-y_{e}^{2}\right] .
$$

that is, the difference in the expectation value of $y^{2}$ for the exciton's electron and hole. Figure $\mathbf{5 f}$ plots such values, from SC to LC NPLs. As the core length increases, the hole is pushed farther away from the center, which translates into a nearly linear increase of $\left\langle y_{h}^{2}\right\rangle$. The electron response is less trivial. In non-interacting e-hh pairs, the increase of the core size should have weak influence, as the electron would stay in the center and only the wave function tail would expand. However, Figure $\mathbf{5} \mathbf{f}$ shows a superlinear increase of $\left\langle y_{e}^{2}\right\rangle$. This is a consequence of the strong Coulomb interaction. As quantum confinement along $y$ becomes weaker, Coulomb attraction drives the electron gradually closer to the core-crown interface, as already seen in panel a (pink curve). It follows that only a moderate increase of $\left\langle y_{e}^{2}-y_{h}^{2}\right\rangle$ takes place with $L_{y}^{c}$. This is consistent with SC and LC NPLs showing similar $S$ values in the experiment.

Because the presence of trapped carriers has been found to affect exciton energy through spectral diffusion, it is also expected to have consequences upon vibronic coupling. The presence of off-centered charges, such as $Q_{i n t}$, reduce the system symmetry and switch on the dipolar contribution in the coupling term $v_{\mu \mu}$ (Figure 6c). However, the fractional surface charge inferred from the energy magnitude of the spectral jumps is small, and its location largely random. One can then expect the intrinsic quadrupolar terms to prevail over the dipolar ones, which would explain the lack of major differences in the Huang-Rhys factor of SC and LC NPLs.

\section{Discussion}

Before concluding we wish to comment further about the obtained results: a comprehensive discussion of the exciton-phonon coupling strength issue in chalcogenide based nanocrystals 
can be found in the work of Groeneveld and Donega. ${ }^{43}$ In good agreement with the latter authors we find that the phonon-mediated transitions are characterized by relatively large Huang-Rhys factors (sometimes larger than unity) with vibronic progressions that look like the ones observed in molecules. This strong coupling is explained by the nature of the interaction mechanisms (of Frölich type) associated to the strong polarizability of the material combined, in type-II systems, to the existence of a permanent dipole associated to the IX. Similar effects were also clearly evidenced and modeled in type-I self-assembled truncated pyramidal quantum dots experiencing a large separation of electron and hole charge clouds. ${ }^{35}$ However we emphasize that in CdSe/CdTe core-crown unperturbed (free of trapped charge) NPLs, the dipolar contribution associated to the electron-hole charge density distribution is null (see above) meaning that higher order contributions may be strong enough to recover a strong exciton-phonon coupling. But we also conjecture that the presence of trapped carriers breaks the charge density symmetry and results in the generation of a permanent dipole in the structure. This contribution, even if moderate, increases the exciton phonon coupling and, as a corollary, enhances the amplitude of spectral diffusion through the linear Stark effect. Our results indicate the high sensitivity of the optical response to the charge felt by the neutral exciton. In our geometry, the net additional charge required to shift the line according to the experimental observations $(|Q|=0.2 e)$ is smaller than the $|Q|=2 e$ that have been estimated to induce similar shifts in type-I dot-in-rod structures. ${ }^{52}$ The small thickness of NPLs as compared to dot-in-rods largely explains the higher sensitivity. One should also remark that, in that work, calculations disregard the surface polarization induced by charges on the (dielectrically mismatched) interface between nanocrystal and ligands.

Correlations could also be expected between the 'instantaneous' vibronic coupling strength (through the Huang-Rhys factor value) and the shift amplitude in the PL emission as it changes in time, the larger the coupling, the larger the PL shift. ${ }^{53}$ Such a strong correlation was not detected in the individual responses (PL traces) in which a relative stability of the spectral shape is quasi-systematically observed, whatever the shift (see for example Figure 
$\mathbf{2 e )}$. This has a strong implication meaning that, in this system, and in line with the analysis of Groeneveld and coworkers, ${ }^{43}$ the exciton-phonon strength revealed in steady-state micro-PL measurements in the frequency domain, is merely of intrinsic nature and cannot be considered as dominated by charge accumulation at the surface. ${ }^{54,55}$ This also means that the quadrupolar term of the bare (non electrostatically perturbed) IX remains the main contribution in front of the dipolar term that charges could generate.

We remark that spectral diffusion probably operates with two different time scales. ${ }^{56}$ The one occuring at second time scales explains the spectral jumps evidenced in Figure 2c and the one leading to the substantial broadening of the ZPL and vibronic satellites that at 5 $\mathrm{K}$ should remain in the few hundreds of $\mu \mathrm{eV}-\mathrm{meV}$ range in the frame of acoustic-phonon mediated broadening in type-II systems. ${ }^{28,33,57,58}$ Though having similar effects in the shift magnitude the latter process should occur at much shorter time scales. ${ }^{59}$ The dynamics of charge fluctuation is out of reach in this study, but we speculate that both processes have an electrostatic origin, as proposed for CdSe/CdS nanocrystals. ${ }^{60}$

\section{Conclusion}

In summary we have studied the low temperature emission properties of prototypical low thickness $(\approx 1.3 \mathrm{~nm})$ type-II core-crown CdSe/CdTe nanoplatelets. By combining theoretical and optical investigations at the single object level we demonstrate that the PL is dominated by the recombination of neutral IX excitons and their phonon replicas, whose binding energy is severely enhanced by dielectric confinement. To reach this conclusion different NPLs designs were used to be able to discriminate between the possible excitations. In agreement with theoretical predictions the PL from IX recombination remains relatively stable as the core dimensions are varied. On the contrary the strong energy shift that would impact charged species (trions) are not observed as the core dimensions change. Calculations also 
allow to rule out Auger-assisted recombination and inter-NPL coupling to explain the mutiple PL peaks, that are the signature of strong exciton LO-phonons coupling. We further show that spectral jumps that show up in the PL recorded in time can be explained by fluctuations of charges residing either at the NPL surface or trapped at the CdSe/CdTe interface. In the explored core-crown geometry a fractionnal charge is sufficient to account for the $\approx 10 \mathrm{meV}$ amplitude of the reported shifts. We conjecture that electrostatic processes of the same type that cannot be resolved in our experiments and that engage similar amount of charge also explain the individual broadening of the ZPL and its vibronic satellites. Beyond spectral diffusion, trapping and detrapping of surface charges can be expected to modify exciton coupling to phonons through increased exciton polarization. However our measurements show that the latter effect can only be of minor importance in the studied system whose exciton-phonon strength (with Huang-Rhys factor around unity) remains intrinsic i.e. quasiuninfluenced by the charged defects. 


\section{Methods}

\section{Synthesis of the 4ML-thick CdSe/CdTe NPLs}

Chemicals. Octadecene (ODE) (Aldrich, $90 \%$ ), cadmium acetate dihydrate (Sigma Aldrich, $98 \%$ ), cadmium oxide (CdO) (Strem, $99.99 \%$ ), myristic acid (Aldrich, > $99 \%$ ), oleic acid (OA) (Aldrich $90 \%$ ), trioctylphosphine (TOP) (Aldrich, $97 \%$ ), selenium (Strem Chemicals 99.99 \%), tellurium (Aldrich, 30 mesh, 99.997 \%), hexane (Carlo Erba, 95 \%), ethanol (Carlo Erba, 99.9 \%).

TOP Te 1M. In a three neck flask, $2.54 \mathrm{~g}$ of Te powder are mixed with $20 \mathrm{~mL}$ of trioctylphosphine and degassed under vacuum at room temperature until the pressure gets reduced below $1 \mathrm{mBar}$. Then the flask is heated under $\mathrm{Ar}$, at $275{ }^{\circ} \mathrm{C}$ until the black $\mathrm{Te}$ powder gets fully dissolved. The orange solution is then cooled and turns yellow. Once the temperature is below $40{ }^{\circ} \mathrm{C}$, the solution is further degassed under vacuum for $10 \mathrm{~min}$ and finally stored in an air-free glove box.

Cadmium Myristate. In a $50 \mathrm{~mL}$ three neck flask, $1.28 \mathrm{~g}$ of $\mathrm{CdO}(10 \mathrm{mmol})$ and 5.5 g of myristic acid $(24 \mathrm{mmol})$ are degassed for 30 minutes at $70{ }^{\circ} \mathrm{C}$. Then, under argon flow, the mixture is heated up to $200{ }^{\circ} \mathrm{C}$ for 30 minutes until it becomes colorless. At the end of the reaction $25 \mathrm{~mL}$ of methanol are added between $60{ }^{\circ} \mathrm{C}$ and $70{ }^{\circ} \mathrm{C}$ in order to solubilize the excess of myristic acid. The cadmium myristate is then precipitated in a centrifuge tube with addition of excess methanol. The washing procedure is repeated at least three times and the cadmium myristate is finally dried overnight under vacuum.

Large (Small) Core Synthesis. In a three neck flask, $340 \mathrm{mg}(\mathbf{2 4 0} \mathbf{~ m g})$ of $\mathrm{Cd}$ (myristate $)_{2}$, $24 \mathrm{mg}$ of Se powder and $25 \mathrm{~mL}$ of octadecene are degassed for 10 minutes under vacuum. Then under argon flow, the mixture is heated up to $240{ }^{\circ} \mathrm{C}$, and when the temperature reaches $205^{\circ} \mathrm{C}, 110 \mathrm{mg}(\mathbf{1 6 0} \mathbf{~ m g})$ of $\mathrm{Cd}(\text { Acetate })_{2}, \mathrm{nH}_{2} \mathrm{O}$ are swiftly introduced. After 10 minutes at $240{ }^{\circ} \mathrm{C}$, the mixture is cooled down to room temperature and $500 \mu \mathrm{l}$ of oleic acid are added. The NPLs are precipitated with a mixture $30 \mathrm{ml}$ of hexane and $30 \mathrm{~mL}$ of ethanol. 
The NPLs are suspended in $20 \mathrm{~mL}$ of hexane.

Core/Crown CdSe/CdTe NPLs Synthesis. ${ }^{16} 3 \mathrm{~mL}$ of large CdSe core NPLs (O.D. of 0.6 at the maximum of the first exciton transition for $100 \mu \mathrm{L}$ of suspension of large CdSe NPLs in $3 \mathrm{~mL}$ of hexane in a $1 \mathrm{~cm}$ length cuvette) are precipitated with ethanol, suspended in $2 \mathrm{~mL}$ of $\mathrm{ODE}$ and transferred in a three neck flask with $24 \mathrm{mg}$ of $\mathrm{Cd}(\mathrm{Ac})_{2}, \mathrm{nH}_{2} \mathrm{O}$ and $45 \mu \mathrm{l}$ of Oleic Acid. The mixture is degassed under vacuum for $15 \mathrm{~min}$, then under argon flow it is heated to $215{ }^{\circ} \mathrm{C}$. At $215{ }^{\circ} \mathrm{C}, 1.5 \mathrm{~mL}$ of a solution containing $3 \mathrm{ml}$ of ODE and 75 $\mu \mathrm{L}$ of TOP Te at $1 \mathrm{M}$ are injected at a rate of $4 \mathrm{~mL} / \mathrm{h}$. Finally, the mixture is cooled to room temperature and washed once with ethanol. The core/crown CdSe/CdTe NPLs are dispersed in hexane and stored in the dark to prevent them from photodegradation.

The same protocol is used for the small core but the quantity of CdSe NPLs is adjusted to have the same amount of CdSe NPLs as for the large ones (in term of surface).

\section{Sample Preparation and Optical Spectroscopy}

Two types of NPLs samples were fabricated and obtained basically using the same dispersion procedure but different concentrations of the original solution. NPLs homogeneous films for absorption and PL measurements were prepared on precleaned (acetone / RBS detergent / distilled water in ultrasonic bath) coverslips substrates $(\approx 0.12 \mathrm{~mm}$ thickness for good thermal contact in cryogenic operations). The concentration was adjusted empirically to reach moderate absorbance at resonance $(0.5 \leq$ Optical Density $\leq 0.8)$. Strong dilution was applied to generate samples for single NPLs studies. This was done according to a "trial and error' protocol in order to reach a dispersion compatible with single NPL selection through far field optical experiments. Typical dilutions around a few $10^{3}$ were required in the present work. All samples were prepared from freshly synthesized material (2 hours following colloidal synthesis at maximum) and transitory kept in an inert Ar atmosphere to prevent oxidative damage before studies. Measurements were systematically performed within 1 day of NPL synthesis. Samples placed in vacuum in the course of cryogenic experiments 
were found stable for a few days typically.

Low temperature absorption spectra were recorded using a Cary 5000 (Varian Agilent) spectrophotometer working in a dual channel mode to get rid of the substrate contribution. The samples were stuck (silver varnish) on the cold finger of a home-made He-flow cryostat allowing $\mathrm{T} \approx 10 \mathrm{~K}$ measurements. The photoluminescence of the dense films was recorded on the micro-PL setup described below, replacing the microscope objective by a short focal length lens $(f=40 \mathrm{~mm})$ to average the PL signal over a larger sample area.

Single NPLs responses were studied on a micro-PL setup working in a confocal-like configuration. The samples are fixed on the cold copper plate of a He-flow micro-PL cryostat designed for thermal expansion compensation (Oxford Instruments); the excitation beam and collected photons are separated with a non-polarizing 70:30(R:T) beamsplitter cube (Thorlabs). The sample surface is imaged onto the entrance slits plane of an imaging spectrometer (Acton SP2760i from Roper Scientific - Princeton Instruments) thanks to an infinity-corrected, 8 $\mathrm{mm}$ working distance, microscope objective $\left(\mathrm{NA}=0.6, f_{e q}=7 \mathrm{~mm}\right)$ attached to a three-axis piezo stage and an achromatic doublet $(f=100 \mathrm{~mm})$. The PL is collected using the same objective and measured following its dispersion (1200 lines/mm, $550 \mathrm{~nm}$ blazed diffraction grating) with a nitrogen-cooled CCD camera (SPEC10, RS - PI) coupled to the spectrometer. A typical 1 micrometer spatial resolution characterizes the setup in routine experiments whereas a $\approx 50 \mu \mathrm{eV}$ energy resolution is reached.

\section{Theoretical Model}

Calculations are carried within $\mathrm{k} \cdot \mathrm{p}$-continuum elastic theory framework. Independent electron and hole states are calculated with single-band Hamiltonians including strain and selfenergy potential terms. Model details and material parameters are given in Ref. ${ }^{61}$ Point charge electrostatic potentials and Coulomb integrals for Configuration Interaction matrix elements, including dielectric mismatch effects, are calculated solving Poisson Equation with Comsol 4.2. 


\section{Acknowledgement}

JP and JIC acknowledge support from MICINN project CTQ2017-83781-P. VS, TB, LL, AC, FB, MC and CT are grateful to Mathieu Bernard and Maxime Vabre for the invaluable support in cryogenics and work in mechanics at INSP. RP and AR acknowledge financial support from the EPSRC and Winton Program for the Physics of Sustainability. RP thanks Alejandro Montblanch, Matteo Barbone, Hannah Stern and Mete Atatüre (Cambridge) for assistance with initial experiments. YP acknowledges the Swedish Research Council for grant VR-2017-05285. AS and GL would like to thank the Australian Research Council for support under Grant CE170100026. SI thanks ERC starting grant Ne2Dem (grant no. 853049).

\section{Supporting Information Available}

The Supporting Information is available free of charge on the ACS Publications website at DOI:Xxx

NPLs dimensions characterization (TEM images); Complementary analyses: role of dielectric mismatch, temperature dependence of the band gap, low temperature Raman spectroscopy and single NPLs PL spectra adjustments (extraction of Huang-Rhys factors).

\section{References}

(1) Lo, S. S.; Mirkovic, T.; Chuang, C.-H.; Burda, C.; Scholes, G. D. Emergent Properties Resulting from Type-II Band Alignment in Semiconductor Nanoheterostructures. Adv. Mater. 2011, 23, 180-197.

(2) Kim, S.; Fisher, B.; Eisler, H.-J.; Bawendi, M. Type-II Quantum Dots: CdTe/CdSe(Core/Shell) and CdSe/ZnTe(Core/Shell) Heterostructures. J. Am. Chem. Soc. 2003, 125, 11466-11467. 
(3) Milliron, D. J.; Hughes, S. M.; Cui, Y.; Manna, L.; Li, J.; Wang, A. P., L-W andAlivisatos Colloidal Nanocrystal Heterostructures with Linear and Branched Topology. Nature 2004, 430, 190-195.

(4) de Mello Donega, C. Formation of Nanoscale Spatially Indirect Excitons: Evolution of the Type-II Optical Character of CdTe/CdSe Heteronanocrystals. Phys. Rev. B: Condens. Matter Mater. Phys. 2010, 81, 165303.

(5) Nanda, J.; Ivanov, S. A.; Marc, A.; Bezel, I.; Piryatinski, A.; Klimov, V. I. Light Amplification in the Single-Exciton Regime Using Exciton-Exciton Repulsion in TypeII Nanocrystal Quantum Dots. J. Phys. Chem. C 2007, 111, 15382-15390.

(6) Li, Q.; Lian, T. Exciton Spatial Coherence and Optical Gain in Colloidal TwoDimensional Cadnium Chalcogenide Nanoplatelets. Acc. Chem. Res. 2019, 52, 26842693.

(7) Kumar, S.; Jones, M.; Lo, S. S.; Scholes, G. D. Nanorod Heterostructures Showing Photoinduced Charge Separation. Small 2007, 3, 1633-1639.

(8) A.V., A.; Prudnikau, A. V.; Melnikau, D.; Rakovich, Y. P.; Chuvilin, A.; Woggon, U.; Achstein, A. W.; Artemyev, M. V. Colloidal Synthesis and Optical Properties of Type-II CdSe-CdTe and Inverted CdTe-CdSe Core-Wing Heteronanoplatelets. Nanoscale 2015, 7, 8084-8092.

(9) Osovsky, R.; Cheskis, D.; Kloper, V.; Sashchiuk, A.; Kroner, M.; Lifshitz, E. Continuous-Wave Pumping of Multiexciton Bands in the Photoluminescence Spectrum of a Single CdTe-CdSe Core-Shell Colloidal Quantum Dot. Phys. Rev. Lett. 2009, 102, 197401.

(10) Oron, D.; Kazes, M.; Banin, U. Multiexcitons in Type-II Colloidal Semiconductor Quantum Dots. Phys. Rev. B: Condens. Matter Mater. Phys. 2007, 75, 035330. 
(11) Klenowski, P.; Steindl, P.; Geffroy, D. Excitonic Structure and Pumping Power Dependent Emission Blue-Shift of Type-II Quantum Dots. Sci. Rep. 2017, 7, 45568.

(12) Park, K. W.; Deutsch, Z.; Li, J. J.; Oron, D.; Weiss, S. Single Molecule QuantumConfined Stark Effect Measurements of Semiconductor Nanoparticles at Room Temperature. ACS Nano 2012, 6, 10013-10023.

(13) Taherkhani, M.; Willatzen, M.; Mørk, J.; Gregersen, N.; McCutcheon, D. P. S. TypeII Quantum-Dot-in-Nanowire Structures with Large Oscillator Strength for Optical Quantum Gates Applications. Phys. Rev. B: Condens. Matter Mater. Phys. 2017, 96, 125408.

(14) Lupo, M. G.; Della Sala, F.; L., C.; Zavelani-Rossi, M.; Fiore, A.; Lüer, L.; Polli, D.; Cingolani, R.; Manna, L.; Lanzani, G. Ultrafast Electron-Hole Dynamics in Core/Shell CdSe/CdS Dot/Rod Nanocrystals. Nano Lett. 2008, 8, 4582-4587.

(15) McDaniel, H.; Heil, P. E.; Tsai, C.-L.; Kim, K.; Shim, M. Integration of Type II Nanorod Heterostructures into Photovoltaics. ACS Nano 2011, 5, 7677-7683.

(16) Pedetti, S.; Ithurria, S.; Heuclin, H.; Patriarche, G.; Dubertret, B. Type-II CdSe/CdTe Core/Crown Semiconductor Nanoplatelets. J. Am. Chem. Soc. 2014, 136, 16430-16438.

(17) Wu, K.; Li, Q.; Jia, Y.; McBride, J. R.; Xie, Z.-X.; Lian, T. Efficient and ultrafast Formation of Long-Lived Charge-Transfer Exciton State in Atomically Thin Cadnium Selenide/Cadnium Telluride Type-II Heteronanosheets. ACS Nano 2015, 9, 961-968.

(18) Kelestemur, Y.; Olutas, M.; Delikanli, S.; Guzelturk, B.; Zafer Akgul, M.; Demir, H. V. Type-II Colloidal Quantum Wells: CdSe/CdTe Core/Crown Heteronanoplatelets. J. Phys. Chem. C 2015, 119, 2177-2185.

(19) Antanovich, A. V.; Prudnikau, A. V.; Melnikau, D.; Rakovich, Y. P.; Chuvilin, A.; Woggon, U.; Achtstein, A. W.; Artemyev, M. V. Colloidal Synthesis and Optical Prop- 
erties of Type-II CdSe-CdTe and Inverted CdTe-CdSe Core-Wing Heteronanoplatelets. Nanoscale 2015, 7, 8084-8092.

(20) Li, Q.; Xu, Z.; McBride, J. R.; Lian, T. Low Threshold Multiexciton Optical Gain in Colloidal CdSe/CdTe Core/Crown Type-II Nanoplatelet Heterostructures. ACS Nano 2017, 11, 2545-2553.

(21) Pandya, R.; Chen, R.; Cheminal, A.; Dufour, M.; Richter, J. M.; Thomas, T. H.; Ahmed, S.; Sadhanala, A.; Booker, E. P.; Divitini, G.; Deschler, F.; Greenham, N. C.; Ithurria, S.; Rao, A. Exciton-Phonon Interactions Govern Charge-Transfer-State Dynamics in CdSe/CdTe Two-Dimensional Colloidal Heterostructures. J. Am. Chem. Soc. 2018, 140, 14097-14111.

(22) Ithurria, S.; Tessier, M. D.; Mahler, B.; Lobo, R.; Dubertret, B.; Efros, A. L. Colloidal Nanoplatelets with Two-Dimensional Electronic Structure. Nature Mater. 2011, 10, 936-941.

(23) Wang, J.-H.; Liang, G.-J.; Wu, K.-F. Long-lived Single Excitons, Trions, and Biexcitons in CdSe/CdTe Type-II Colloidal Quantum Wells. Chin. J. Chem. Phys. 2017, 30, 649656.

(24) Grim, J. Q.; Christodoulou, S.; Di Stasio, F.; Krahne, R.; Cingolani, R.; Manna, L.; Moreels, I. Continuous-wave biexciton lasing at room temperature using solutionprocessed quantum wells. Nature Nanotechnology 2014, 9, 891-895.

(25) Benchamekh, R.; Gippius, N. A.; J., E.; Nestoklon, M. O.; Jancu, J.-M.; Ithurria, S.; Dubertret, B.; Efros, A. L.; P., V. Tight-Binding Calculations of Image-Charge Effects in Colloidal Nanoscale Platelets of CdSe. Phys. Rev. B: Condens. Matter Mater. Phys. 2014, 89, 035307.

(26) Scott, R.; Achtstein, A. W.; Prudnikau, A. V.; Antanovich, A.; Siehbeles, L. D. A.; Artemyev, M.; Woggon, U. Time-Resolved Stark Spectroscopy in CdSe Nanoplatelets: 
Exciton Binding Energy, Polarizability, and Field-Dependent Radiative Rates. Nano Letters 2016, 16, 6576-6583.

(27) Rajadell, F.; Climente, J. I.; Planelles, J. Excitons in Core-Only, Core-Shell and Core-Vrown CdSe Nanoplatelets: Interplay Between In-Plane Electron-Hole Correlation, Spatial Confinement, and Dielectric Confinement. Phys. Rev. B: Condens. Matter Mater. Phys. 2017, 96, 035307.

(28) Krivolapchuk, V. V.; Moskalenko, E. S.; Zhmodikov, A. I. Specific Features of the Indirect Exciton Luminescence Line GaAs/ $\mathrm{Al}_{x} \mathrm{Ga}_{1-x} \mathrm{As}$ Double Quantum Wells . Phys. Rev. B: Condens. Matter Mater. Phys. 2001, 64, 045313.

(29) Scholes, G. D.; Jones, M.; Kumar, S. Energetics of Photoinduced Electron-Transfer Reactions Decided by Quantum Confinement. J. Phys. Chem. C 2007, 111, 1377713785.

(30) Kim, S.; Fisher, B.; Eisler, H.-J.; Bawendi, M. Type-II Quantum Dots: CdTe/CdSe(Core/Shell) and CdSe/ZnTe(Core/Shell) Heterostructures. J. Am. Chem. Soc. 2003, 125, 11466-11467.

(31) Pandya, R. et al. Fine Structure and Spin Dynamics of Linearly Polarized Indirect Excitons in Two-Dimensional CdSe/CdTe Colloidal Heterostructures. ACS Nano 2019, 13, 10140-10153.

(32) Dufour, M.; Steinmetz, V.; Izquierdo, E.; Pons, T.; Lequeux, N.; Lhuillier, E.; Legrand, L.; Chamarro, M.; Barisien, T.; Ithurria, S. Engineering Bicolor Emission in 2D Core/Crown CdSe/CdSe ${ }_{1-x} \mathrm{Te}_{x}$ Nanoplatelet Heterostructures Using Band-Offset Tuning. J. Phys. Chem. C 2017, 121, 24816-24823.

(33) Fernée, M. J.; Tamarat, P.; Lounis, B. Spectroscopy of Single Nanocrystals . Chem. Soc. Rev. 2014, 43, 1311-1337. 
(34) Tessier, M. D.; Javaux, C.; Maksimovic, I.; Loriette, V.; Dubertret, B. Spectroscopy of Single CdSe Nanoplatelets. ACS Nano 2012, 6, 6751-6758.

(35) Kindel, C.; Callsen, G.; Kako, S.; Kawano, T.; Oishi, H.; Hönig, G.; Schliwa, A.; Hoffmann, A.; Arakawa, Y. Spectral Diffusion in Nitride Quantum Dots: Emission Energy Dependent Linewidths Broadening Via Giant Built-In Dipole Moments. Phys. Status Solidi RRL 2014, 8, 408-413.

(36) Henderson, B.; Imbusch, G. Optical Spectroscopy of Inorganic Solids; Oxford University Press, 2006.

(37) Antolinez, F. V.; Rabouw, F. T.; Rossinelli, A. A.; Cui, J.; Norris, D. J. Observation of Electron Shakeup in CdSe/CdS Core/Shell Nanoplatelets. Nano Lett. 2019, 19, 84958502.

(38) Movilla, J. L.; Planelles, J.; Climente, J. I. Dielectric Confinement Enables Molecular Coupling in Stacked Colloidal Nanoplatelets. The Journal of Physical Chemistry Letters 2020, 11, 3294-3300.

(39) Scott, R.; Heckmann, J.; Prudnikau, A. V.; Antanovich, A.; Mikhailov, A.; Owschimikow, N.; Artemyev, M.; Climente, J. I.; Woggon, U.; Grosse, N. B.; Achtstein, A. W. Directed Emission of CdSe Nanoplatelets Originating from Strongly Anisotropic 2D Electronic Structure. Nature Nanotechnology 2017, 12, 1155-1161.

(40) Rodina, A.; Efros, A. L. Effect of Dielectric Confinement on Optical Properties of Colloidal Nanostructures. J. Exp. Theor. Phys. 2016, 122, 554-566.

(41) Achtstein, A. W.; Antanovich, A.; Prudnikau, A.; Scott, R.; Woggon, U.; Artemyev, M. Linear Absorption in CdSe Nanoplates: Thickness and Lateral Size Dependency of the Intrinsic Absorption. J. Phys. Chem. C 2015, 119, 20159-20161. 
(42) Feng, F.; Nguyen, L.; Nasilowski, M.; Nadal, B.; Dubertret, B.; Coolen, L.; Maitre, A. Consequence of Shape Elongation on Emission Asymmetry for Colloidal CdSe/CdS Nanoplatelets. Nano Res. 2018, 11, 3593-3602.

(43) Groeneveld, E.; de Mello Donegá, C. Enhanced Exciton-Phonon Coupling in Colloidal Type-II CdTe-CdSe Heteronanocrystals. J. Phys. Chem. C 2012, 116, 16240-16250.

(44) Wang, S.; Wang, L. W. Exciton Dissociation in CdSe/CdTe Heterostructure Nanorods. J. Phys. Chem. Lett. 2011, 2, 1-6.

(45) Scott, R.; Kickhöfel, S.; Schoeps, O.; Antanovich, A.; Prudnikau, A.; Chuvilin, A.; Woggon, U.; Artemyev, M.; Acthstein, A. W. Temperature Dependent Radiative and Non-Radiative Recombination Dynamics in CdSe-CdTe and CdTe-CdSe Type II Hetero Nanoplatelets. Phys. Chem. Chem. Phys. 2016, 18, 3197-3203.

(46) Shornikova, E. V. et al. Addressing the Exciton Fine Structure in Colloidal Nanocrystals: the Case of CdSe Nanoplatelets. Nanoscale 2018, 10, 646-656.

(47) Yu, J.; Zhang, C.; Pang, G.; Sun, X. W.; Chen, R. Effect of Lateral Size and Surface Passivation on the Near-Band-Edge Excitonic Emission from Quasi-Two-Dimensional CdSe Nanoplatelets. ACS Applied Materials 83 Interfaces 2019, 11, 41821-41827.

(48) Di Stasio, F.; Imran, M.; Akkerman, Q. A.; Prato, M.; Manna, L.; Krahne, R. Reversible Concentration-Dependent Photoluminescence Quenching and Change of Emission Color in $\mathrm{CsPbBr}_{3}$ Nanowires and Nanoplatelets. J. Phys. Chem. Lett. 2017, 8, $2725-2729$.

(49) Planelles, J.; Achtstein, A. W.; Scott, R.; Owschimikow, N.; Woggon, U.; Climente, J. I. Tuning Intraband and Interband Transition Rates via Excitonic Correlation in LowDimensional Semiconductors. ACS Photonics 2018, 5, 3680-3688. 
(50) Califano, M.; Franceschetti, A.; Zunger, A. Temperature Dependence of Excitonic Radiative Decay in CdSe Quantum Dots: The Role of Surface Hole Traps. Nano Letters 2005, 5, 2360-2364.

(51) Nomura, S.; Kobayashi, T. Exciton LO-Phonon Couplings in Spherical Semiconductor Microcrystallites. Phys. Rev. B: Condens. Matter Mater. Phys. 1992, 45, 1305-1316.

(52) Lohmann, S. H.; Strelow, C.; Mews, A.; Kipp, T. Surface Charges on CdSe-Dot/CdSRod Nanocrystals: Measuring and Modeling the Diffusion of Exciton-Fluorescence Rates and Energies. ACS Nano 2017, 11, 12185-12192.

(53) Empedocles, S. A.; Bawendi, M. G. Quantum-Confined Stark Effect in Single CdSe Nanocrystallite Quantum Dots. Science 1997, 278, 2114-2117.

(54) Sagar, D. M.; Cooney, R. R.; Sewall, S. L.; Dias, E. A.; Barsan, M. M.; Butler, I. S.; Kambhampati, P. Size Dependent, State-Resolved Studies of Exciton-Phonon Couplings in Strongly Confined Semiconductor Quantum Dots. Phys. Rev. B: Condens. Matter Mater. Phys. 2008, 77, 235321.

(55) Kambhampati, P. Hot Exciton Relaxation Dynamics in Semiconductor Quantum Dots: Radiationless Transitions on the Nanoscale. J. Phys. Chem. C 2011, 115, 22089-22109.

(56) Fernée, M. J.; Plakhotnik, T.; Louyer, Y.; Littleton, B. N.; Potzner, C.; Tamarat, P.; Mulvaney, P.; Lounis, B. Spontaneous Spectral Diffusion in CdSe Quantum Dots. J. Phys. Chem. Lett. 2012, 3, 1716-1720.

(57) Shafran, E.; Borys, N. J.; Huang, J.; Talapin, D. V.; Lupton, J. M. Indirect Exciton Formation Due to Inhibited Carrier Thermalization in Single CdSe/CdS Nanocrystals. J. Chem. Phys. Lett. 2013, 4, 691-697.

(58) Beian, M.; Alloing, M.; Cambril, E.; Gomez Carbonell, C.; Osmond, J.; Lemaître, A.; 
Dubin, F. Long-lived Spin Coherence of Indirect Excitons in GaAs Coupled Quantum Wells. EPL 2015, 110, 27001.

(59) Coolen, L.; Brokmann, X.; Spinicelli, P.; Hermier, J.-P. Emission Characterization of a Single CdSe-ZnS Nanocrystal with High Temporal and Spectral Resolution by Photon Correlation Fourier Spectroscopy. Phys. Rev. Lett. 2008, 100, 027403.

(60) Beyler, A. P.; Marshall, L. F.; Cui, J.; Brokmann, X.; Bawendi, M. G. Direct Observation of Rapid Discrete Spectral Dynamics in Single Colloidal CdSe-CdS Core-Shell Quantum Dots. Physical Review Letters 2013, 111, 177401.

(61) Llusar, J.; Planelles, J.; Climente, J. I. Strain in Lattice-Mismatched CdSe-Based Core/Shell Nanoplatelets. The Journal of Physical Chemistry C 2019, 123, 2129921306. 


\section{Graphical TOC Entry}

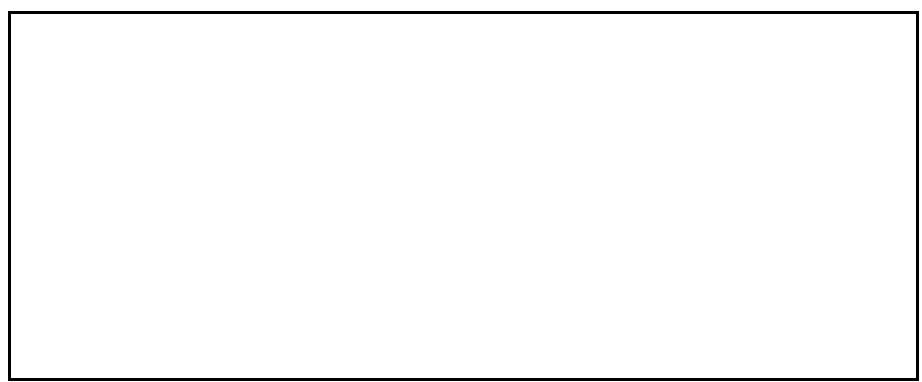

Size (as specified in the JPC C Author Guidelines for Table of Contents/Abstract Graphics): $8.25 \mathbf{~ c m ~} \times$ $4.45 \mathrm{~cm}$.

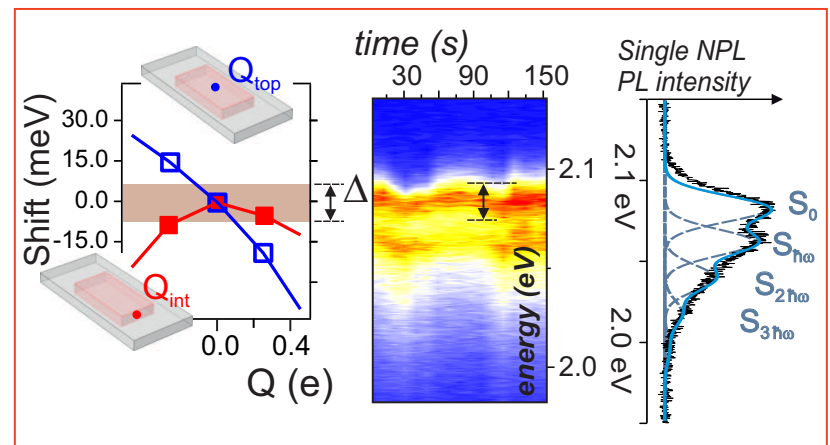




\title{
Emission State Structure and Linewidth Broadening Mechanisms in Type-II CdSe/CdTe Core-Crown Nanoplatelets: A Combined Theoretical - Single Nanocrystal Optical Study
}

\section{Supporting Information}

\author{
Violette Steinmetz, ${ }^{\dagger}$ Juan I. Climente ${ }^{\ddagger}{ }^{\ddagger}$ Raj Pandya, ${ }^{\circledR}$ Josep Planelles, ${ }^{\ddagger}$ Florent \\ Margaillan, ${ }^{\dagger}$ Yuttapoom Puttisong, ${ }^{\S}$ Marion Dufour, " Sandrine Ithurria," Ashish \\ Sharma, ${ }^{\perp}$ Girish Lakhwani, ${ }^{\perp}$ Laurent Legrand, ${ }^{\dagger}$ Frédérick Bernardot,${ }^{\dagger}$ Christophe \\ Testelin $^{\dagger}{ }^{\dagger}$ Maria Chamarro, ${ }^{\dagger}$ Alex W. Chin ${ }^{\dagger}$ Akshay Rao, ${ }^{\top}$ and Thierry Barisien ${ }^{*} \dagger$ \\ †Sorbonne Université, Institut des NanoSciences de Paris, INSP, CNRS-UMR 7588, 4 \\ place Jussieu, F-75005, Paris, France
}

$\ddagger$ Departament de Química Física i Analítica, Universitat Jaume I, E-12080, Castelló de la Plana, Spain

IDepartment of Physics, Cavendish Laboratory, University of Cambridge, 19 JJ Thompson Avenue, Cambridge, CB3 OHE, UK

$\S$ Functional Electronic Materials, Department of Physics, Chemistry and Biology, Linköping University, 58183 Linköping, Sweden

||Laboratoire de Physique et d'Étude des matériaux, ESPCI Paris, PSL Research University, CNRS UMR 8213, 10 rue Vauquelin, 75005 Paris, France $\perp A R C$ Centre of Excellence in Exciton Science, School of Chemistry, The University of Sydney, Sydney, New South Wales 2006, Australia

E-mail: barisien@insp.jussieu.fr 


\section{Electron microscopy, temperature dependence of the band}

\section{gap, effect of dielectric contrast and low temperature Ra-}

\section{man spectroscopy}

\section{T.E.M. characterization}

(a)

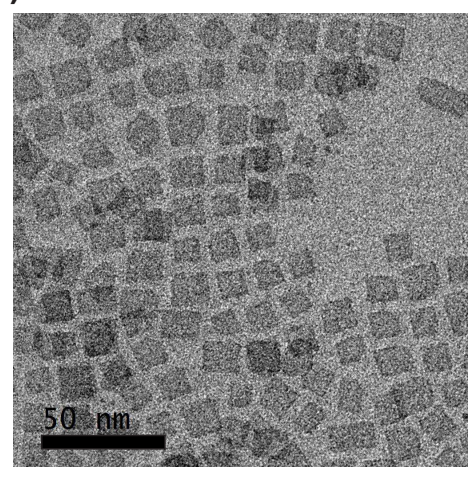

(c)

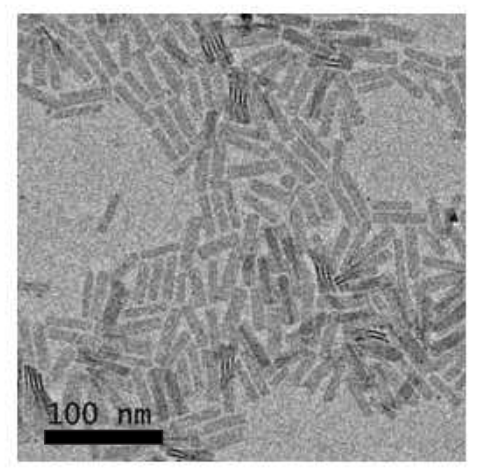

(b)

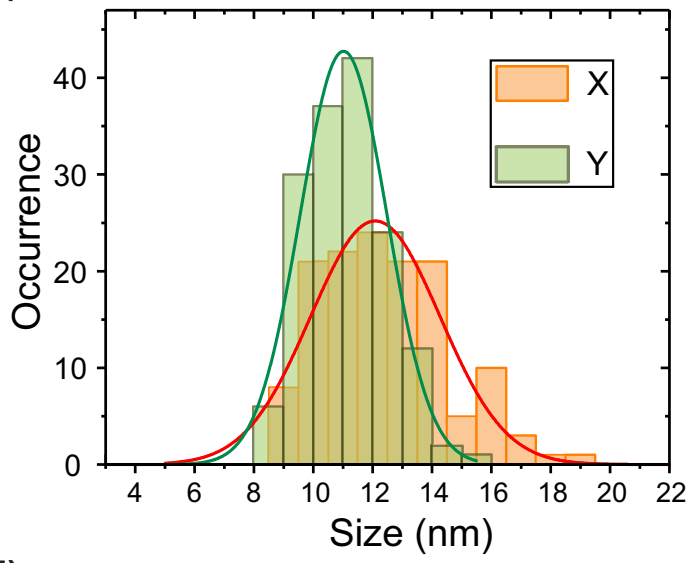

(d)

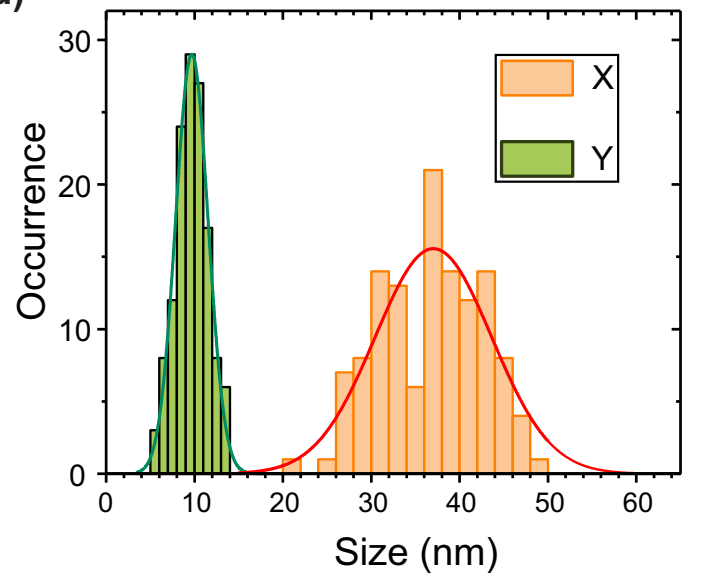

Fig. S. 1: Average NPLs core sizes extracted from TEM images analysis. (a) and (c): TEM images of core structures before crown growing leading to SC and LC NPLs respectively. (b) and (d): size distributions are adjusted using normal distributions - using two images for each of the species - leading to average sizes $\left\langle X_{S C}\right\rangle=12.0 \mathrm{~nm}($ FWHM $=5.2 \mathrm{~nm}),\left\langle Y_{S C}\right\rangle=11.0 \mathrm{~nm}$ $(\mathrm{FWHM}=3.4 \mathrm{~nm})$ and $\left\langle X_{L C}\right\rangle=37.0 \mathrm{~nm}(\mathrm{FWHM}=15.5 \mathrm{~nm}),\left\langle Y_{L C}\right\rangle=9.7 \mathrm{~nm}(\mathrm{FWHM}=$ $4.3 \mathrm{~nm})$. We note the substantial amount of dispersity in the explored samples. 


\section{Temperature dependence of the band gap}

A strong shift of the excitonic transitions, $X_{i}$, towards higher energies is observed as the temperature is lowered. The measured variations are typical of II/VI semiconductors. We check here that they can indeed be predicted by the semiempirical Varshny relation that describes the behaviour of the band gap, $E_{g}$, with temperature: ${ }^{1,2}$

$$
E_{g}(T)=E_{g}(0)-\frac{\alpha T^{2}}{\beta+T} .
$$

The equation 1 is built in order to account for the variation of two contributions in the energy gap:

- At high temperature the relative position of the conduction band and valence band is due to the dilatation of the lattice. Theoretical calculations show the effect is linear with T.

- The electrons-lattice interaction (major contribution) also changes the gap; this leads to distinct dependences: $\Delta E_{g} \propto T$ above the Debye temperature and $\Delta E_{g} \propto T^{2}$ below the Debye temperature.

$X_{1}$ and $X_{2}$ transitions correspond to the lowest e-hh transition in CdTe and CdSe respec-

tively. Their shifts are plotted in figure 2. Adjustments of the curves provide $\alpha$ and $\beta$ values in close agreement with the reported values ${ }^{2}$ except for the $X_{2}$ transition (CdSe) in large crown NPLs where the totally unconstrained fit leads to a significantly smaller $\beta$ parameter. We notice that the quality of the fit poorly depends on $\beta$ in a wide range of the parameter value. We find:

- for SC NPLs:

$$
\begin{aligned}
& E_{g, C d T e}(0) \approx 2.337 \pm 0.004 \mathrm{eV} ; \alpha_{C d T e} \approx 0.57 \pm 0.13 \mathrm{meV} / \mathrm{K} \text { and } \beta_{C d T e} \approx 166 \pm 107 \mathrm{~K} ; \\
& E_{g, C d S e}(0) \approx 2.535 \pm 0.003 \mathrm{eV} ; \alpha_{C d S e} \approx 0.62 \pm 0.22 \mathrm{meV} / \mathrm{K} \text { and } \beta_{C d S e} \approx 265 \pm 202 \mathrm{~K} ; \\
& \text { - for LC NPLs: } \\
& E_{g, C d T e}(0) \approx 2.333 \pm 0.003 \mathrm{eV} ; \alpha_{C d T e} \approx 0.62 \pm 0.17 \mathrm{meV} / \mathrm{K} \text { and } \beta_{C d T e} \approx 199 \pm 133 \mathrm{~K} ; \\
& E_{g, C d S e}(0) \approx 2.545 \pm 0.004 \mathrm{eV} ; \alpha_{C d S e} \approx 0.50 \pm 0.10 \mathrm{meV} / \mathrm{K} \text { and } \beta_{C d S e} \approx 114 \pm 78 \mathrm{~K} ;
\end{aligned}
$$



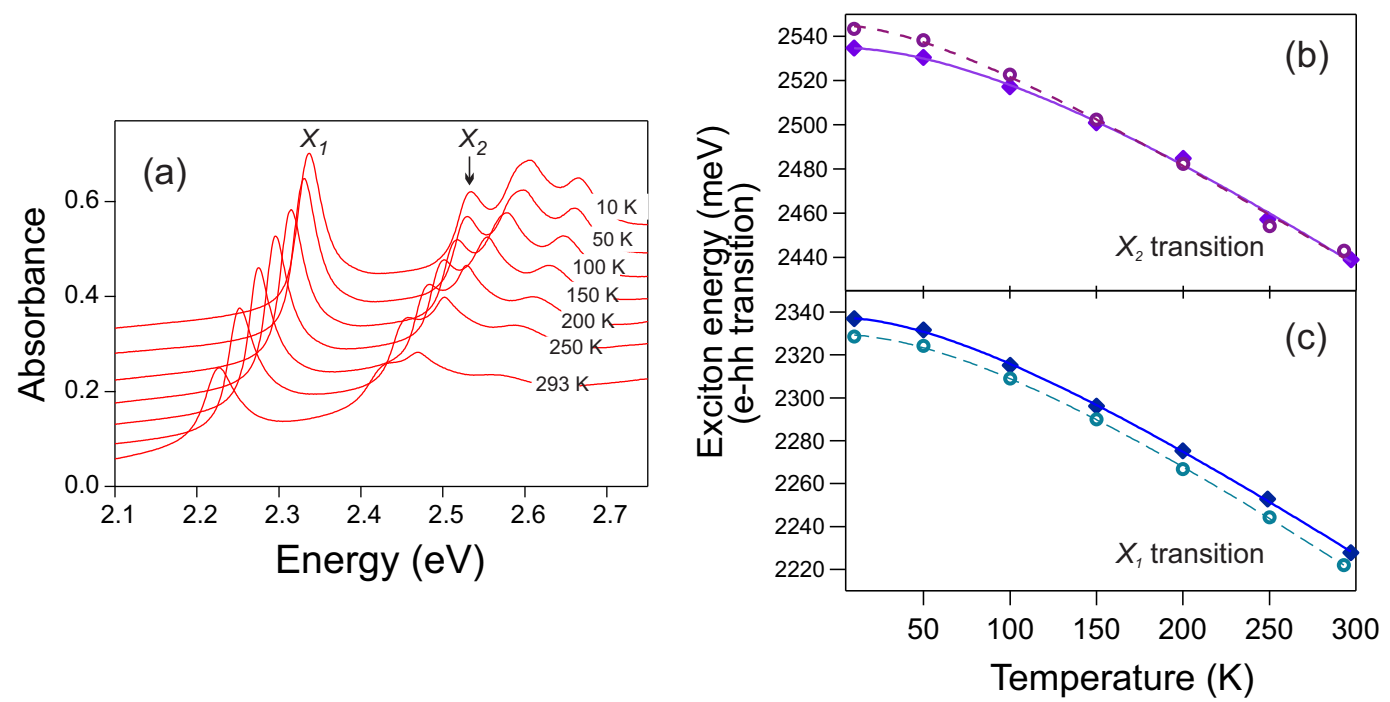

Fig. S. 2: (a) Absorption as a function of temperature (10 K - $293 \mathrm{~K})$ in small core (SC) NPLs showing the characteristic blue shift as $T$ decreases. (b) Energy of the $X_{2}$ resonance (CdSe core) in small core (SC) NPLs (solid diamond) and large core (LC) NPLs (open circle). (c) Energy of the $X_{1}$ resonance (CdTe crown) - legend as in (b).

Reducing the uncertainty on $\alpha$ and $\beta$ parameters would require improved measurements (with reduced uncertainty on $T$, improved spectral resolution and larger set of values). Accurate modelling of the gap evolution in NPLs should in addition account for the confined nature of phonons, a task beyond the scope of the present study. We provide here a rough estimation of the Varshny parameters for 4 monolayers core-crown NPLs having different core sizes and emphasize that the $\approx 100 \mathrm{meV}$ shift observed between room temperature and $10 \mathrm{~K}$ is typical of line displacements reported so far in II/VI semiconductors heterostructures. We finally notice that the smooth evolutions of the $X_{1}$ and $X_{2}$ transition energies are very likely the indication that there is no phase-transition occuring in the system as temperature varies between $4 \mathrm{~K}$ and $300 \mathrm{~K}$.

\section{Effect of dielectric contrast}

In core-crown CdSe/CdTe NPLs the fine structure of indirect excitons was found to be composed of two linearly polarized states associated to dipoles contained in the NPL plane and having orthogonal orientations. ${ }^{3}$ Due to the large difference in the dielectric constant of the 
CdSe and CdTe semiconductor materials and the surrounding medium (vacuum in our low $\mathrm{T}$ experiments) the electric field penetrating the NPL is reduced. This local field effect drastically depends on the nanocrystal geometry and changes the photon coupling to an exciton. ${ }^{4}$ In order to reach a realistic estimation of the field modification in a simplified picture, NPLs are modelled as flattened homogeneous ellipsoids of revolution $\left(\epsilon_{C d S e} \approx \epsilon_{C d T e}=\epsilon_{i n}, \epsilon\right.$ being the relative permittivity) characterized by the semi-axis length $a, b$ and $c$ (in the $X, Y$ and $Z$ directions respectively).

The projection of the electric field inside, $E_{\alpha}^{\text {in }}$, and outside, $E_{\alpha}^{\text {out }}$, the nanocrystal $(\alpha=X$, $Y, Z)$ are related as:

$$
E_{\alpha}^{\text {in }}=\frac{E_{\alpha}^{\text {out }}}{1+n^{\alpha}(k-1)}
$$

where $k=\epsilon_{\text {in }} / \epsilon_{\text {out }}\left(\epsilon_{\text {out }}=1\right)$ and $n^{\alpha}$ is the depolarisation factor along the direction $\alpha$. Calculations by Landau and Lifshitz provide: ${ }^{5}$

$$
n^{\alpha}=\frac{1}{2} a b c \int_{0}^{\infty} \frac{d s}{\left(s+\beta^{2}\right) \sqrt{\left(s+a^{2}\right)\left(s+b^{2}\right)\left(s+c^{2}\right)}}
$$

with $\beta=\mathrm{a}($ for $\alpha=X)$, b $(\alpha=Y)$ or c $(\alpha=Z)$.

As the exciting field is tuned far away the excitonic resonance the local field effect is considered in the emission process only. The probability to emit light for each level composing the fine structure is reduced by the screening factor, $D_{\alpha}=\left(E_{\alpha}^{\text {in }} / E_{\alpha}^{\text {out }}\right)^{2}$. $^{4}$

In LCs NPLs, numerical integration of equation 3 with $a=65 \mathrm{~nm}, b=18 \mathrm{~nm}, c=1.3 \mathrm{~nm}$, leads to:

$$
\begin{aligned}
& \text { - } n^{X}=0.0093 \text { and } n^{Y}=0.0638\left(\text { with } \epsilon_{\text {in }} / \epsilon_{\text {out }} \approx 10\right) ; \\
& \text { - } \frac{D_{X}}{D_{Y}} \approx 2
\end{aligned}
$$

In SCs NPLs,we find, with $a=33 \mathrm{~nm}, b=19 \mathrm{~nm}, c=1.3 \mathrm{~nm}$ :

- $n^{X}=0.0247$ and $n^{Y}=0.0557$;

- $\frac{D_{X}}{D_{Y}} \approx 1.5$. 


\section{Low temperature Raman spectroscopy}

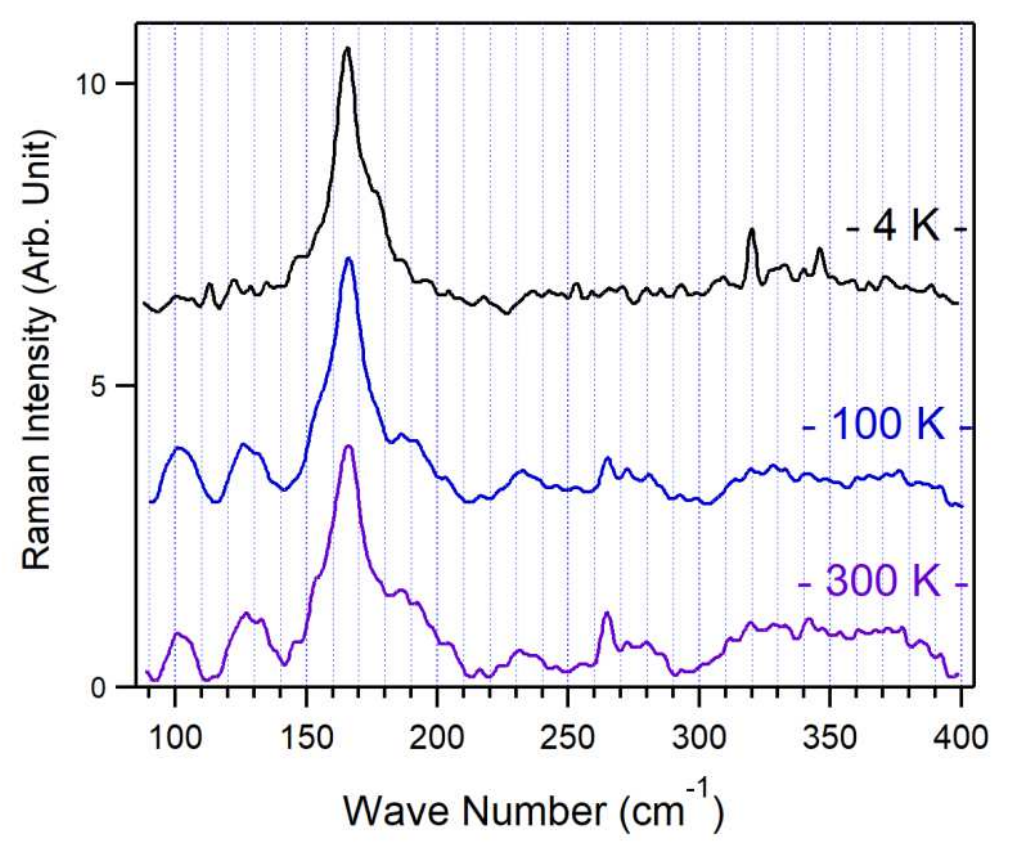

Fig. S. 3: Raman spectrum measured at three temperatures (from top to bottom: $4 \mathrm{~K}, 100 \mathrm{~K}$ and $300 \mathrm{~K}$ ) in CdSe/CdTe core-crown NPLs films (moderate dilution). Raman measurements were conducted by backscattering (T64000, Horiba) a CW diode line (532 nm, $1 \mathrm{~mW}$ ) with a triple stage. Spectra were collected at $>80 \mathrm{~cm}^{-1}$, where the CCD detector (Horiba Synapse Open-Electrode) has a monotonically increasing quantum efficiency of $0.43-0.50$. Acquisitions employed a $100 \times$ optical objective and used minimal laser intensity to avoid sample degradation. The spectrum is dominated by a single peak which position does not move as temperature changes. We report a Raman shift of $166 \pm 2 \mathrm{~cm}^{-1}(\Delta E=20.6 \pm 0.3 \mathrm{meV})$ in agreement with the periodic modulation of single NPLs spectra.

\section{Huang-Rhys factor extraction}

Following the approach of Groeneveld and Donega ${ }^{6}$ the PL lineshape may be modelled as a sum comprising a pure electronic line (zero phonon line or ZPL) and several secondary components peaked at lower energy, (i) each associated to a phonon-assisted emission process (phonon replicae) and, (ii) each displaced in energy by an amount corresponding to multiples of the energy of the phonon involved in the transition. For a transition between the excited excitonic (b) state toward the electronic (a) state, the emission lineshape as a function of 
the energy, $E$, reads (keeping the notation of Groeneveld and coworker ${ }^{6}$ ):

$$
I_{a b}(E)=I_{0} \sum_{n} F_{n}(0) \delta\left(E_{0,0}-n \hbar \omega-E\right)
$$

where $E_{0,0}=E_{b, 0}-E_{a, 0}$ is the energy of the transition between the zero vibrationnal levels of the initial (b) state and final (a) state (ZPL) and $n$ is the number of phonons generated in the transition. $I_{0}$ is the intensity of the full band and $F_{n}(0)$ is the $T=0$ Franck-Condon factor and can be expressed as: ${ }^{7}$

$$
F_{n}(0)=\frac{\exp (-S) S^{n}}{n !}
$$

$S$ being the Huang-Rhys parameter. We note that up to three phonons can be observed in the PL of single core-crown NPLs.

Equation 4 is in priniple only valid in the frame of the adiabatic approximation stating that the electron-phonon coupling does not give rise to transition between different electronic configurations which could be observed when there are degenerate states or electronic states with energy spacings comparable to phonon energies. The lattice motion is a coupled system problem however the description in terms of displacements of individual ions can be reduced to a single configurational coordinate model (using normal coordinates) in the harmonic approximation (like in molecular systems). ${ }^{7}$ The electron-phonon interaction Hamiltonian is then taken to be linear in the normal vibrationnal amplitude. The ZPL has the intensity $I_{0} \exp (-S)$ and if $S=0$ all the intensity is contained in the ZPL. As $S$ increases the intensity in the ZPL decreases and this is compensated for by the emergence of vibrational sidebands at energies $n \hbar \omega$ below the ZPL.

Extraction of the Huang-Rhys factor: the single NPLs spectra were adjusted using the progression given by equations 4 and 5 . The $n^{\text {th }}$ vibronic line has a weight $I_{0} F_{n}(0)$ in the band, which is extracted from the adjustment of the experimental curve (see below for procedure). Plotting $F_{n+1}(0) / F_{n}(0)$ as a function of $(n+1)^{-1}$ allows to estimate the value of $S$ (slope of 
the plot). When doing so, we are led to the following conclusions:

- The expected linear dependence is indeed observed in the majority of considered objects (see figure 4.a-c, e);

- Some deviations are however reported and show up in the basic adjustment of single NPLs spectra (see for instance fit of figure 4.d);

- $0.8<S<1$ is found in more than $80 \%$ of the studied nanocrystals; we report spectra characterized by $S>1$ but the occurrence is much less frequent;

- Some of the spectra cannot be adjusted reasonably using the progression of equation $4(\approx 10 \%)$ and it was decided they would not contribute to the statistics presented in the study.

Guidelines for the adjustment protocol: the sharpest peaks at higher energy (ZPL and first phonon replica) are first considered and provide most of the guess values injected in the fit:

(1) From their positions in energy we extract the spacing (phonon energy) $\Delta E_{1}=$ $E_{a, 1}-E_{a, 0}=\hbar \omega_{1}, \Delta E_{2}=\hbar \omega_{2}, \ldots \Delta E_{p}$. When peaks do not show up for $p>2$, the transition energies, $E_{n}$, were generated as follows: $E_{n}=E_{b, 0}-E_{a, n} \approx E_{0,0}-n \hbar \omega_{2}$;

(2) The ratio of the amplitudes provides a first estimation of the Huang-Rhys factor, $S=F_{1} / F_{0}$. As a first step the weighting coefficients, $F_{n}$, are imposed by equation 5 . They can be further optimized through the fit procedure, as a second step.

(3) As a first approximation correct fits are obtained by imposing identical linewidths for all phonon sidebands. It is however clear that some of the ZPLs are found to have slightly narrower linewidths. 

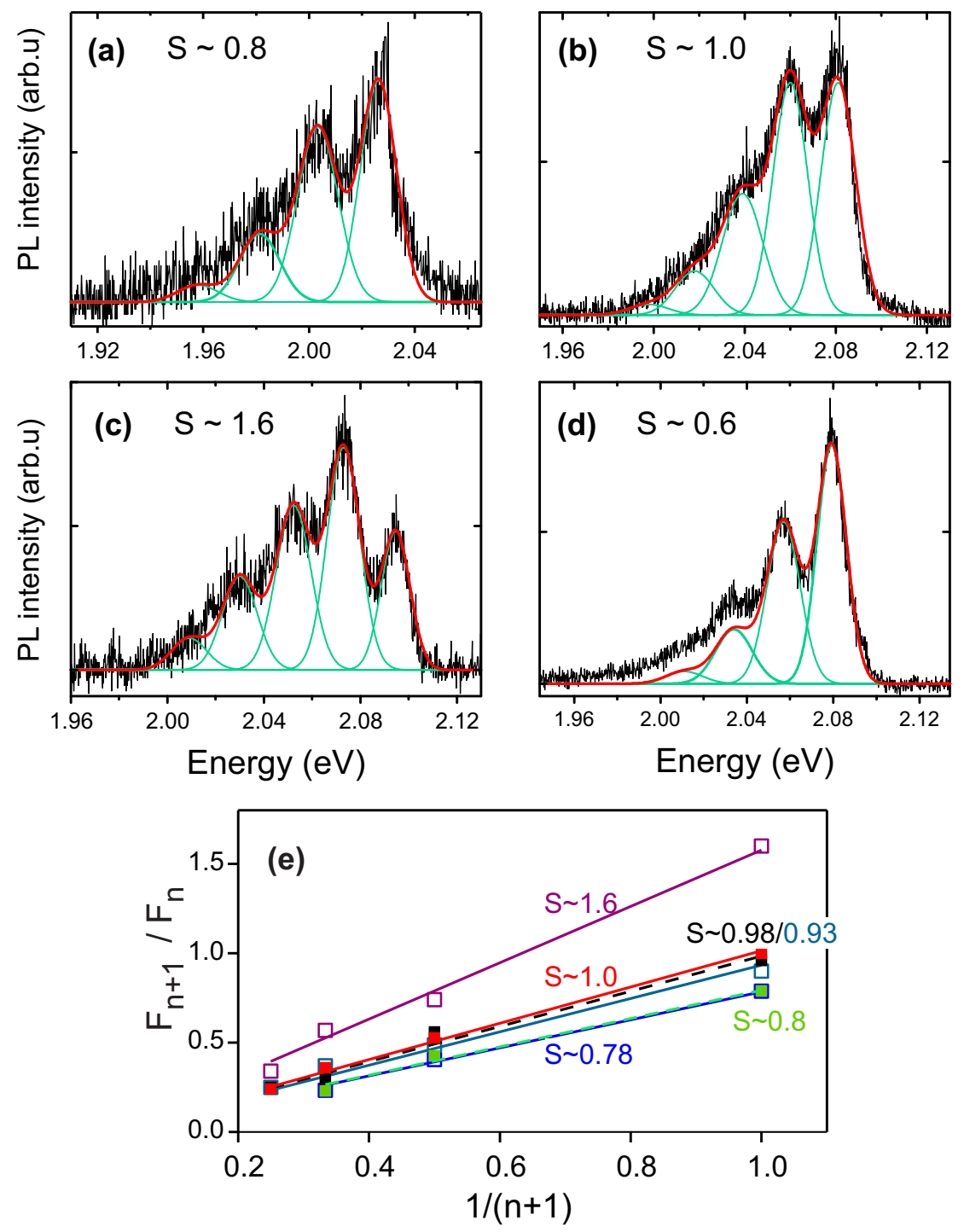

Fig. S. 4: Typical PL spectra from single CdSe/CdTe core-crown NPLs with different HuangRhys factors: (a) $S \approx 0.8$, (b) $S \approx 1.0$, (c) $S \approx 1.6$ and (d) $S \approx 0.6$; in the latter case the adjustment, based on the ZPL - first replica amplitude ratio, is clearly less good, showing a slight underestimation of the fit on the low energy side. Most of the studied NPLs ( $\geq 80 \%$ in number) had a Huang-Rhys factor around $0.8-1$. The green curves represent the components (ZPL and phonon replicae) from which the relative amplitude $F_{n}$ are extracted (see text). (e) Plot of the $F_{n+1} / F_{n}$ ratio as a function of $(n+1)^{-1}$ in six NPLs. The linear dependence is expected for a phonon progression in the displaced oscillator model; the slope provides the Huang-Rhys factor, $S$.

\section{References}

(1) Varshny, Y. P. Temperature Dependence of the Energy Gap in Semiconductors. Physica 1967, 34, 149-154. 
(2) Tamargo, M., Ed. II-VI Semiconductor Materials and their Applications; Optoelectronic Properties of Semiconductors and Superlattices; CRC Press, 2002; Vol. 12.

(3) Pandya, R. et al. Fine Structure and Spin Dynamics of Linearly Polarized Indirect Excitons in Two-Dimensional CdSe/CdTe Colloidal Heterostructures. ACS Nano 2019, 13, $10140-10153$.

(4) Rodina, A.; Efros, A. L. Effect of Dielectric Confinement on Optical Properties of Colloidal Nanostructures. J. Exp. Theor. Phys. 2016, 122, 554-566.

(5) Landau, L.; Lifshitz, E. M. Course of theoretical Physics, Vol. 8: Electrodynamics of Continuous Media; Pergamon: Oxford, 1960.

(6) Groeneveld, E.; de Mello Donegá, C. Enhanced Exciton-Phonon Coupling in Colloidal Type-II CdTe-CdSe Heteronanocrystals. J. Phys. Chem. C 2012, 116, 16240-16250.

(7) Henderson, B.; Imbusch, G. Optical Spectroscopy of Inorganic Solids; Oxford University Press, 2006. 